\title{
Two-dimensional nanochannel membranes for molecular and ionic separations
}

Shaofei Wang, $\dagger^{\text {ad }}$ Leixin Yang, $\dagger^{\text {ab }}$ Guangwei He, ${ }^{\text {ae }}$ Benbing Shi, ${ }^{\text {ab }}$ Yifan Lidf , Hong Wu, ${ }^{\text {ab }}$ Runnan Zhang, ${ }^{\text {ab }}$ Suzana Nunes ${ }^{\star d}$, and Zhongyi Jiang ${ }^{\star a b c}$

a Key Laboratory for Green Chemical Technology of Ministry of Education, School of Chemical Engineering and Technology, Tianjin University, Tianjin 300072, China. Email: zhyjiang@tju.edu.cn

${ }^{b}$ Collaborative Innovation Center of Chemical Science and Engineering (Tianjin), Tianjin 300072, China.

c Joint School of National University of Singapore and Tianjin University, International Campus of Tianjin University, Binhai New City, Fuzhou, 350207, China.

d Biological and Environmental Science and Engineering Division (BESE), Advanced Membranes and Porous Materials Center (AMPM), King Abdullah University of Science and Technology (KAUST), Thuwal, 23955-6900, Saudi Arabia. E-mail: suzana.nunes@kaust.edu.sa.

e Institute of Chemical Sciences and Engineering (ISIC), École Polytechnique Fédérale de Lausanne (EPFL), Sion, Switzerland.

${ }^{f}$ School of Chemical Engineering and Energy, Zhengzhou University, Zhengzhou 450001, PR China.

$\dagger$ These authors contributed equally to this work

\section{Abstract}

Two-dimensional (2D) nanosheets have emerged as promising functional materials owing to the atomic thickness and unique physical/chemical properties. By using 2D nanosheets as the building blocks, diverse kinds of two-dimensional nanochannel membranes (2DNCMs) are being actively explored, in which mass transport occurs in the through-plane and interlayer channels of 2D nanosheets. The rational construction and physical/chemical microenvironments regulation of nanochannels are of vital significance for translating these $2 \mathrm{D}$ nanosheets into molecular separation membranes and ionic separation membranes. Focusing on the recent advances of 2DNCMs, in this review, various porous/nonporous 2D nanosheets and their derived nanochannels are first briefly introduced. Then we discuss the emerging top-down and bottom-up methods to synthesize high-quality 2D nanosheets and to prepare high-performance 2DNCMs. As the major part of this review, we concern three types of nanochannels, which are based on nonporous nanosheets, intrinsically porous nanosheets and perforated nanosheets. The strategies of regulating physical and chemical microenvironments in the nanochannels are emphasized. The representative applications of 2DNCMs in molecular separations (gas 
separation, liquid separation), and ionic separations are presented. Finally, the current challenges and future perspectives are highlighted.

\section{Key learning points}

1. The survey on two-dimensional nanochannel membranes (2DNCMs) for molecular and ionic separations.

2. Top-down and bottom-up methods to synthesize 2D nanosheets as the membrane building blocks.

3. A variety of methods to prepare single-layer and lamellar 2DNCMs.

4. The strategy of regulating nanochannel physical and chemical microenvironments in 2DNCMs.

5. The relationships among nanochannel structure, mass transport mechanism and separation performance of 2DNCMs.

\section{Introduction}

Efficient separation processes to obtain clean water, gases or solvents, which currently account for a significant portion (10-15\%) of the world's energy consumption, are highly demanded in energy and environment-related applications. ${ }^{1}$ Membrane separation technology, as an energy-efficient technology, has been successfully applied in seawater desalination, wastewater treatment, carbon capture, etc. ${ }^{2}$ As the core part, membrane materials with high permeability, high selectivity and high stability are extremely desired since they can significantly accelerate the practical applications of membrane separation technology. ${ }^{3}$ Accordingly, the transmembrane channels, either composed of the fractional free-volume from dense polymer films, the nanopores from semi-rigid polymeric structures or the permanent pores from crystalline frameworks, become the research focus in membrane technology field. ${ }^{2,4}$

Generally, ideal separation membrane channels should integrate the following attributes: (i) they should be short and of high-density to endow membrane with high permeability, (ii) the channel size distribution should be narrow to endow membrane with high selectivity, and (iii) the chemical and mechanical stability should high to endow membrane with long-term stability. ${ }^{5}$ Currently, polymers are the dominant membrane materials, attributing to their easy processability and high scale-up capability. For conventional polymers, breaking the permeability-selectivity trade-off remains a challenge. ${ }^{3}$ The great progress of polymeric membranes over the 50 years has brought about the booming of novel kinds of structured membranes, e.g. mixed matrix membranes, facilitated transport membranes, block-copolymer 
membranes, semi-rigid polymer membranes including thermally rearranged (TR) polymer, polymers of intrinsic microporosity (PIMs)). ${ }^{3,4}$ With a well-regulated free-volume or nanopores, the separation performances of polymer membranes have been pushed to new records. However, preventing the semi-rigid nanopores from swelling or collapse to inhibit plasticization or aging proves not an easy task. ${ }^{4}$ Polycrystalline membranes based on porous framework materials (e.g. metal-organic frameworks (MOF) and covalent organic frameworks (COFs)) impart a more robust channel for precise sieving. Yet, the scalable and controllable crystal growth to fabricate thin and defect-free membrane has been recognized as a grand bottleneck. Therefore, membrane materials that can be easily processed into high-performance separation membranes are imperatively required for sustainable membrane development.

In recent years, two-dimensional (2D) nanosheets, with a thickness of one to a few atoms, have become the promising building blocks for advanced nanochannel membranes. ${ }^{6}$ Although the history of layered materials has been more than 100 years, 2D nanosheets started to gain widespread attention just following the successful exfoliation of graphene, an atomic-scale hexagonal lattice made of carbon atoms, by Novoselov and Geim in 2004. ${ }^{7}$ The 2D nanosheets are quite competitive in various applications ${ }^{8}$ including supercapacitors, optoelectronics, catalysts, chemical/biological sensors, spintronics, and more importantly, in membrane separations, where an ultrathin separation layer is highly demanded. The 2D nanosheets have opened a new era of membranes due to their atomic thickness and unique structures, which can theoretically afford ultimate permeation properties. ${ }^{9}$ Moreover, 2D nanosheets can be designed with precise channel size along with targeted chemical functionalities, enabling their extraordinary physical or chemical selectivities. ${ }^{10}$ Furthermore, $2 \mathrm{D}$ nanosheets can be readily fabricated into membranes by using well-established methods, such as casting or coating, affording the excellent processability. ${ }^{11}$

When the 2D nanosheets are fabricated into membranes, one ideal geometry is single layered membranes, where a highly porous 2D nanosheet attaches on the top of a robust and porous support. ${ }^{12}$ In these membranes, the through-plane, ultrashort channels allow for ultrafast permeation. However, the synthesis of large-sized, defect-free nanosheets confronts many difficulties. ${ }^{13}$ Another geometry, lamellar membranes, made from multilayer stackings, pave a more practical way to large-scale membrane preparation. ${ }^{14}$ Lamellar membranes can be fabricated from either porous or nonporous nanosheets. The mass transport takes place in both the through-plane channels (including the intrinsic/perorated pores in the nanosheet and the intraplanar gaps from the in-plane neighbouring nanosheets) and the interlayer channels. For 
the above-mentioned "nanochannel membranes", since the mass transport and separation occur in the through-plane and/or interlayer nanochannels that are formed by the 2D nanosheets, we define these systems as 2D nanochannel membranes (2DNCMs) to highlight the intrinsic "2D geometry" and "nanochannel membrane" feature. Such definition could also establish the explicit relationship between materials, structures, and membrane performance.

The selective nanochannels in 2DNCMs could originate from nonporous nanosheets, intrinsically porous nanosheets and perforated nanosheets. Nanochannels based on nonporous nanosheets refer to the interlayer nanochannels from the lamellar membranes. When the nanosheets are parallelly stacked, the layer-to-layer repulsive interactions or intercalated compounds will create empty interlayer channels for mass transport. The nonporous nanosheets include graphene oxide (GO), hexagonal boron nitride ( $h$-BN), MXenes, transition metal dichalcogenides (TMDs), layered double hydroxides (LDHs), etc. The interlayer channel is usually tortuous. To shorten the transmembrane distance, it is necessary to generate throughplane channels. ${ }^{15}$ Nanochannels based on intrinsically porous nanosheets refer to the channels from the intrinsic pores of nanosheets. Intrinsically porous nanosheets can be 2D zeolites, 2D MOFs, 2D COFs, graphyne and graphitic carbon nitride $\left(g-\mathrm{C}_{3} \mathrm{~N}_{4}\right)$, etc. Nanochannels based on perforated nanosheets refer to the post-made pores from 2D nanosheets. Theoretically, either porous or nonporous nanosheets can be further physically treated by electron/ion beam or chemically treated via oxidation, plasma, to obtain 2D nanosheets with increased porosity. ${ }^{9}$

Up to now, there have been numerous simulation calculations, experimental exploitations as well as several excellent reviews on membranes based on 2D nanosheets. ${ }^{6,14,16}$ Most of the reviews are material-oriented. Structure-oriented reviews that concentrate on the structure features of 2DCNMs are scarce. Meanwhile, a fast development of 2DCNMs has been witnessing over the past five years due to the evolution of novel chemistry and advanced processing techniques. More precise separation, even down to the angstrom scales, have been achieved. Accordingly, this Tutorial Review focusing on the recent advancement of nanochannel construction and regulation in 2DNCMs may offer a unique perspective. Due to the limited space, mixed matrix membranes using 2D nanosheets as the filler will not be covered here but can be found in some other recent reviews. ${ }^{16}$ The mass transport mechanism and some simulation calculation pertaining to the nanochannels can also be referred to a comprehensive review. ${ }^{5}$ Focusing on the nanochannels, our review covers the nanosheet synthesis, 2DNCMs preparation, nanochannel regulation and membrane applications (Fig.1). We first introduce the top-down and bottom-up methods to synthesize $2 \mathrm{D}$ nanosheets as the 
building blocks. Then the membrane preparation methods, also the channel assembly methods, are introduced for single layer and lamellar membranes. As the major part of this review, the channel construction and microenvironments regulation methods are thoroughly discussed, in terms of the nanochannels from (i) nonporous, (ii) intrinsically porous, and (iii) perforated 2D nanosheets. Noted that, the membrane preparation and nanochannel regulation are sometimes interrelated. To segregate, the membrane preparation section discusses the universal strategies to assemble 2DNCMs, and the nanochannel regulation section focuses on the strategies to tune three different kinds of nanochannels. Subsequently, the application of these 2DNCMs in the field of gas molecular separations, liquid molecular separations and ionic separations are discussed. Finally, we conclude by highlighting the pendent challenges and predicting the future research directions of 2DNCMs. Since channel regulation is the primary focus for all twodimensional material membranes, it can be expected that this review may offer some guidance on the design of diverse nanochannels for the applications in separation, catalysis, energy conversion and storage, etc.

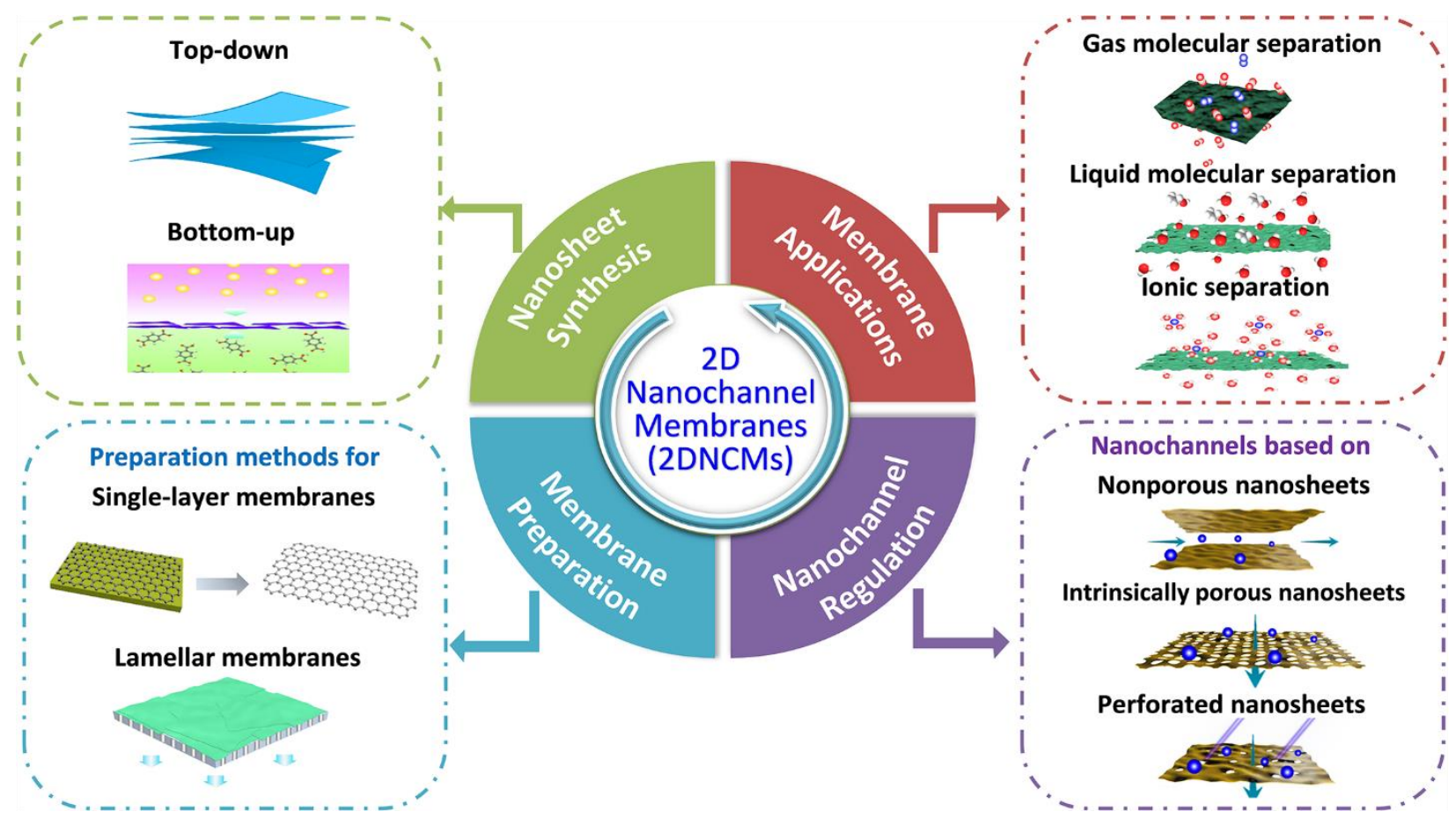

Fig. 1 The scope of this review. This review covers four major aspects of the 2DNCMs, in the sequence of nanosheet synthesis, membrane preparation, nanochannel regulation, and the applications.

\section{Preparation of 2D nanosheets and 2D nanochannel membranes (2DNCMs)}


A high-performance 2DNCM requires high-quality 2D nanosheets as the building blocks, and the delicate membrane preparation strategy to construct the channels. Therefore, special requirements are concentrated on the size, thickness, defects and surface functionalities of the nanosheets. These parameters remarkably affect the selection of membrane preparation methods. A more comprehensive review on the synthesis of broad 2D materials can be found by Tan et al. ${ }^{8}$ In this section, keeping to the nanosheets requirements to be applied in 2DNCMs, we introduce and compare the top-down and bottom-up synthesis methods. Then, the membrane preparation methods, pertaining to the single-layer membrane and lamellar membranes, are discussed.

\subsection{Synthesis of 2D nanosheets}

The synthesis strategy for 2D nanosheets can be generally classified as top-down and bottom-up methods (Fig. 2). The top-down method refers to the exfoliation of 2D nanosheets from the bulk, while the bottom-up method refers to the direct synthesis of 2D nanosheets from the basic building units. As the ideal building blocks, the nanosheets should be only a singlelayer to minimize the mass transport tortuosity. The size of the nanosheets should be large to reduce the number of less-selective intraplanar gaps. ${ }^{13,17}$ Moreover, nanosheets bearing functional groups that facilitate the selective transport should favour the separation applications. $^{18}$

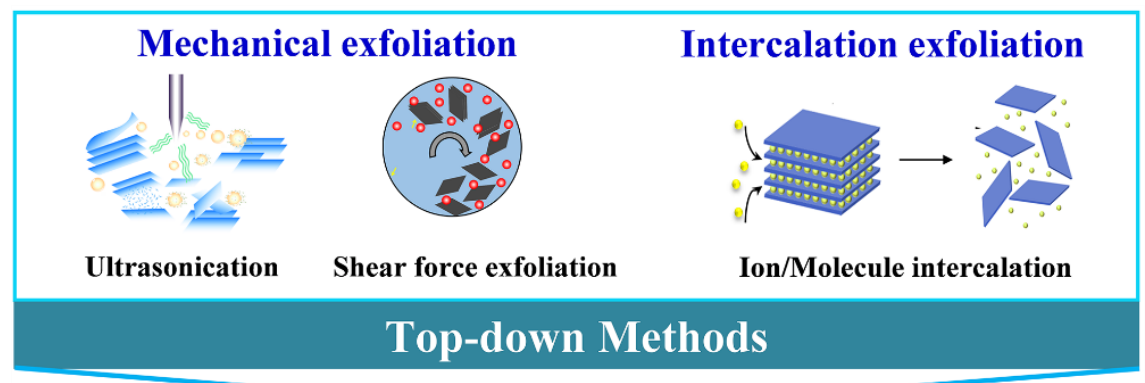

2D Nanosheets

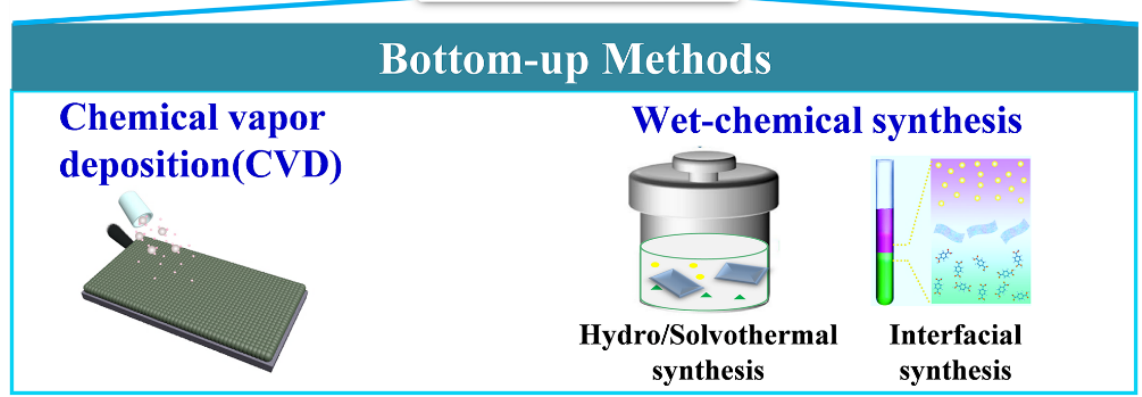

Fig. 2 Representative top-down and bottom-up methods to synthesize 2D nanosheets.

\section{(a) Top-down methods}


Most layered materials are formed by the stacking of monolayers along the vertical direction. The weak interlayer interactions (van der Waals forces, $\pi-\pi$ stacking, hydrogen bonding, etc.) enable a facile exfoliation by a top-down method. The top-down methods can be classified into mechanical and intercalation exfoliations.

The mechanical exfoliation, by using a mechanical force to exfoliate nanosheets, is an easyhandling method. The original microcleavage exfoliation of bulk layered crystals, known as the "Scotch-tape" method, is still in use to prepare highly crystalline single- and few-layer nanosheets, to investigate their intrinsic physical properties. ${ }^{7}$ However, this method suffers from the drawback of low throughput. Nowadays, the more widely adopted approaches are the ultrasonication and shear force exfoliations. These methods are facile to operate, insensitive to environmental conditions, and easily scalable. ${ }^{8}$ After being treated by the above methods, the colloidally stable nanosheets typically comprise a small number of stacked layers (less than 10) that should be further separated by successive centrifugation to yield single-layered 2D nanosheets. ${ }^{19}$ In these processes, besides the parameters like sonication power, rotation rates and processing duration, a judicious solvent selection is also critical for successful exfoliation. The solvent molecular size and hydrophilicity are the major considerations. The pioneering work by Yang's group ${ }^{17}$ achieved the successful exfoliation and membrane application of 1-nm-thick MOF sheets with large lateral size $(\sim 1.5 \mu \mathrm{m})$ and also high crystallinity (Fig. 3). A combined method of low-energy wet ball milling and ultrasonication was used to exfoliate the layered MOFs material, $\mathrm{Zn}_{2}(\mathrm{bim})_{4}$ (bim=benzimidazole). The use of a methanol and $\mathrm{n}$-propanol mixture as the solvent play key role in the successful exfoliation. The smaller molecule, methanol, could diffuse into the MOFs interlayers to assist the exfoliation, whereas n-propanol was used to stabilize the exfoliated nanosheets by attaching onto the surface of the MOFs nanosheets via the hydrophobic alkane chains. This co-solvent strategy should also be effective in other exfoliation methods.

The intercalation exfoliation always accompanies the entrance of ions or molecules into the layers, either to weaken the layer interactions or to improve the surface charge to facilitate the dispersion in solvents. As a typical example, ions (e.g., $\mathrm{Li}^{+}, \mathrm{Na}^{+}$or $\mathrm{K}^{+}$) can intercalate into TMDs via forced hydration, leading to exfoliated nanosheets. MoS 2 is usually obtained by such method. ${ }^{20}$ Though the intercalation exfoliation method can render highly stable dispersion of nanosheets in solvents, the processing time is always long (up to 3 days), a smaller-sized flake $(<1 \mu \mathrm{m})$ is usually required to allow the fluent diffusion of ions into the interlayers. ${ }^{8}$ The oxidation $^{\circ}$ of graphite and subsequent exfoliation in a liquid with the aid of ultrasonication, using Hummers' 
methods or its variations, also belongs to the intercalation exfoliation method. Such method acquires dispersible GO in polar solvents that can be used for large-scale membrane preparation. ${ }^{11}$

Top-down methods are widely used due to the easy operations. These resultant solutionprocessable nanosheets can facilitate the subsequent blending, casting and functionalization for target membrane applications. The intercalation exfoliation method can readily obtain singlelayer nanosheets, but the intercalators used are either explosive or highly oxidative chemicals, which require scrupulous operations. Besides, the residual chemicals on the nanosheets may affect the final performance of membranes, which should be removed by successive washing.

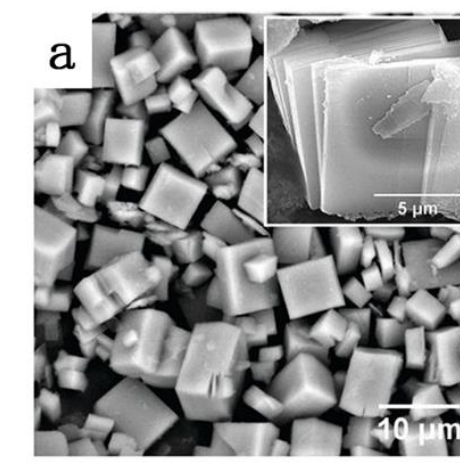

1. Wet Ball Milling

2. U1trasonication

Solvent:

methanol \& n-propanol
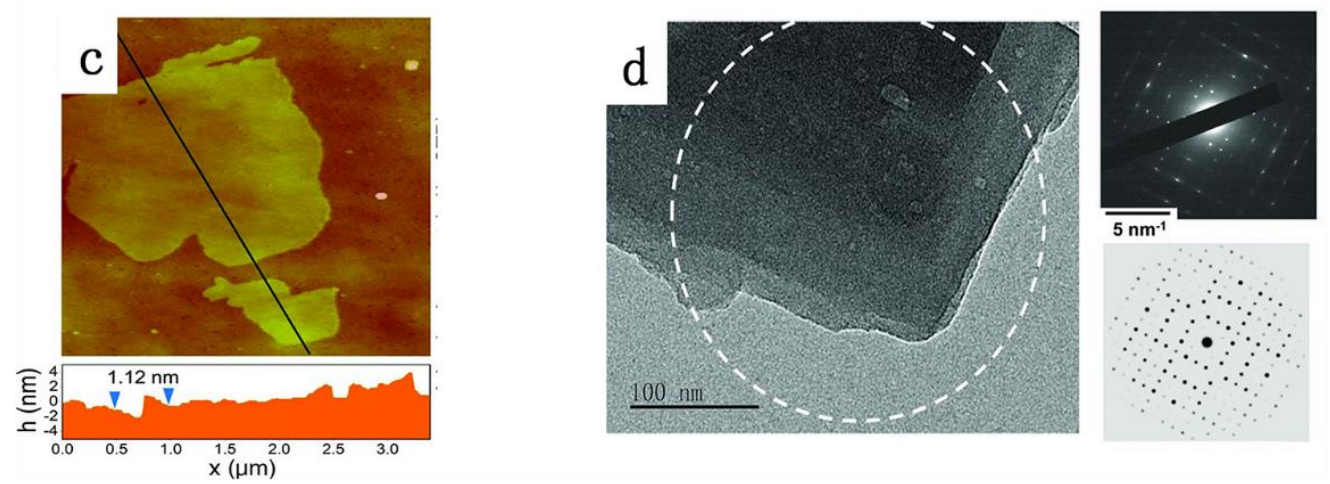

Fig. 3 A top-down method to exfoliate MOF nanosheets. (a) Scanning electron microscopy (SEM) image of $Z n_{2}(\text { bim })_{4}$ crystals. (b) Transmission electron microscopy (TEM) image of the nanosheets. The inset shows the Tyndall effect of a colloidal suspension in a mixed solvent. (c) Atomic force microscope (AFM) image of the nanosheet, indicating a thickness of $\sim 1 \mathrm{~nm}$. (d) Selected area electron diffraction pattern (white circle) shows the diffraction from (hk0) planes within a few-layered nanosheet. The experimental pattern (right up) is similar to the simulated one (right down), meaning a high crystallinity. Reproduced with permission from ref. ${ }^{17}$. Copyright 2014, Science Publishing Group.

\section{(b) Bottom-up methods}


The top-down method can be only applicable when there exists a layered bulk material as the precursor, whereas, the bottom-up method is applicable to almost all 2D materials. Bottom-up methods mainly encompass chemical vapor deposition (CVD) growth and wet-chemical synthesis. The epitaxial growth on substrates (e.g., $\mathrm{SiC}$ and $\mathrm{SiO}_{2}$ ), by CVD, is a well-established method to fabricate graphene, TMDs and $h$-BN. The CVD method allows producing highly crystalline 2D nanosheets with scalable size and controllable thickness, potentially being used as membrane materials.

For typical porous 2D nanosheets (e.g., zeolite, MOF and COFs), made from nods and linkers, the wet-chemical synthesis is the dominant method. The synthesis process is similar to that of the bulk materials, and the success lies in how to selectively block the growth of materials along one direction. ${ }^{21}$ The hydro/solvothermal synthesis and interfacial synthesis are the most popular strategies.

The hydro/solvothermal synthesis, which is often conducted under high temperature and high-pressure conditions, have been used to prepare various nanosheets including 2D metal oxide, metal chalcogenides, MOFs, etc. ${ }^{8}$ Surfactants or structure-directing agents are often introduced to facilitate the formation of nanosheets rather than bulk materials. The thickness, size, and crystallinity of the products can be controlled by altering the synthesis parameters, including the precursor concentration, surfactant and solvent type, reaction temperature/time. Recently, directing the growth of 2D nanosheets by introducing extra agents has been proved effective. Mordenite framework inverted (MFI)-type zeolites are widely attempted as membrane materials but are quite defect-sensitive. Synthesizing single MFI nanosheets via the bottom-up method may eliminate the formation of non-selective structural defects. However, the undesired orthogonal intergrowths (MFI twins) may also occur during the synthesis. The first success was achieved by Tsapatsis's group. ${ }^{21}$ (Fig. 4(a)). The ingenious use of a growth directing agent, bis1,5(tripropyl ammonium) pentamethylene diiodide (dC5), guided the formation of plate-like MFI with the thin crystal dimension along the $b$ axis, producing micrometer-sized MFI nanosheets with a thickness of $5 \mathrm{~nm}$ (2.5 unit cells). The resulting morphology showed abundant straight micropores orthogonal to the sheet surface, conferring the great potential of the application as membranes.

The interfacial synthesis is applicable in both nanosheets and thin membranes. In this method, the reaction between different monomers occurs at the confined interfaces to guarantee the low thickness. The interfaces include liquid/liquid, liquid/air or liquid/solid interfaces. The diffusion 
rates of the monomers towards the interface govern the structural integrity of the produced nanosheets, ${ }^{22}$ which can be modulated by setting an intermediate layer. The instructive MOF synthesis strategy proposed by Rodenas et al. ${ }^{23}$ had displayed the benefit of using multiple liquid layers (Fig. 4(b)). Unlike traditional strategies that grow MOFs at the organic/aqueous interface, their synthesis medium consisted of three liquid layers composed of mixtures of $\mathrm{N}, \mathrm{N}$ dimethyl formamide (DMF) and a suitable miscible co-solvent at appropriate ratios, which were vertically arranged according to their different densities. The top layer and bottom layer had the metal $\left(\mathrm{Cu}\left(\mathrm{NO}_{3}\right)_{2}\right)$ and organic ligand (1,4-benzenedicarboxylic acid (BDC)), respectively. The MOF grew at the intermediate region, which was a highly diluted medium to achieve the thin MOF layers with a lateral size of 0.5-4 $\mu \mathrm{m}$ and thicknesses in the range 5-25 nm. By replacing the corresponding units, this generic synthesis approach can also be extended to produce other types of MOFs nanosheets. Noted that, the interfacial synthesis is usually conducted under a mild condition that might not be sufficient for reactions requiring a high activation energy, like COFs synthesis. By using a Lewis acid, $\mathrm{Sc}(\mathrm{OTf})_{3}$, as the catalyst, Dichtel's group ${ }^{24}$ for the first time realized the room temperature COF synthesis via the interfacial polymerization of a polyfunctional amine (1,3,5-tris(4-aminophenyl)benzene (TAPB)) and a aldehyde monomer (terephthalaldehyde aldehydes (PDA)) at the liquid-liquid interface. The catalyst is critical to facilitate the imine exchange process, which directs the initially formed amorphous network into long-range ordered COF structure. Unlike other methods that separate the monomers into two individual phases, they put both monomers into the organic phase, while keeping the catalyst in the aqueous phase. The spatial segregation of the catalyst and the monomers induced siteselective polymerization, generating continuous COFs films with a thickness as thin as $2.5 \mathrm{~nm}$. However, for practical applications, a further transfer onto a robust and porous substrate is needed. A moderate adhesion between the selective layer and the substrate is essential to meet the requirements of stable performances. 


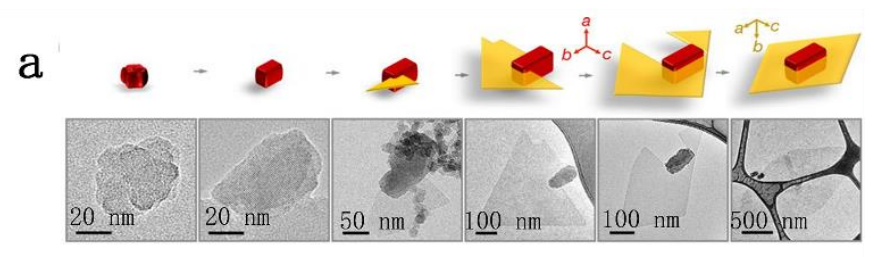

b

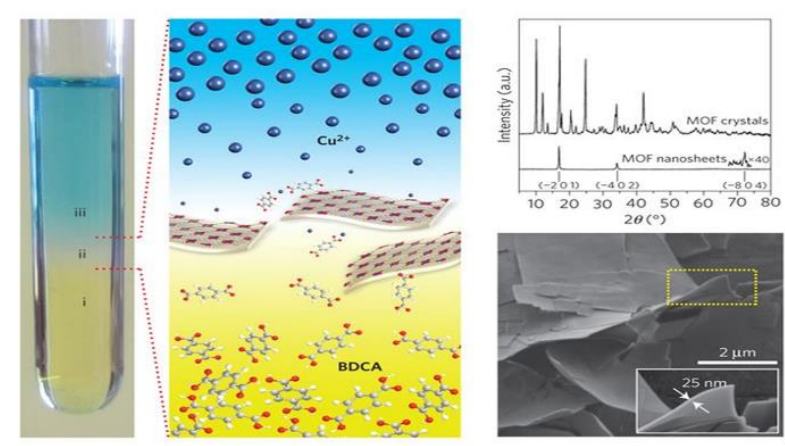

Fig. 4 Bottom-up methods to synthesize 2D nanosheets. (a) A modulated synthesis to direct the formation of plate-like MFI. The top images show the growth mechanism by using the seed crystal, the bottom images show the corresponding TEM morphology. Reproduced with permission from ref. ${ }^{21}$. Copyright 2017, Springer Nature. (b) Interfacial synthesis of CuBDC nanosheets. The left part illustrates the spatial arrangement of different liquid layers. The right part shows the X-ray diffraction (XRD), SEM results of the produced MOF sheets. Reproduced with permission from ref. ${ }^{23}$. Copyright 2015, Springer Nature.

Generally, free of the strong forces to generate fragmentations or morphological damages, the bottom-up method could produce large-sized nanosheets with fewer defects, but the scalability potential is usually lower than the top-down method. Notably, the CVD method allows for the preparation of single-layer membranes. However, the introduction of uniform, highdensity nanopores into the CVD-derived nanosheets proves very difficult. The interfacial synthesis is a facile method to synthesize single to few-layer thin films, of which the lateral size depends on the size of reaction vials. When a porous support is set at the interface, this method provides a straightforward way to prepare composite membranes.

\subsection{Preparation of 2D nanochannel membranes (2DNCMs)}

\subsubsection{Preparation of single-layer membranes}

Single-layer film hosting nanopores is the thinnest membrane and therefore regarded as the ultimate membranes. However, the experimental realization of single-layer membrane remains a huge challenge due to the difficulties in the crack-free transfer of large-area nanosheets and the controllable pore generation (refer to the discussion in Section 3.3 for perforated 
nanosheets). ${ }^{9,13}$ Considering the high mechanical strength and potential of scalable fabrication, porous graphene is screened as the ideal material for single-layer membranes. However, cracks, tears or wrinkles usually evolve when the graphene is transferred on a porous support, deteriorating its separation performance. A frequently adopted solution is the coating of a reinforcement polymer on graphene, e.g. polymethyl methacrylate (PMMA) to improve the mechanical strength. However, the non-permeable polymer has to be removed subsequently, the removal process inevitably brings about cracks. Instead of using polymers, using robust, porous support materials that have a preferential affinity towards graphene is more effective for the large-area transfer. Agrawal's group ${ }^{25}$ applied nanoporous carbon as the mechanical reinforcement, producing the first gas-selective graphene membrane (Fig. 5(a)). The pyrolysis of a block copolymer film, coated on the graphene, could generate nanoporous carbon that had strong interactions with the CVD graphene. The crack-free transfer yielded millimeter-scale single-layer graphene with $\mathrm{H}_{2}$-selective pores.

The size of graphene membranes can be further pushed to centimeter scale by adopting novel-structured supports. Very recently, Duan and coworkers ${ }^{13}$ used a 1D carbon material, an interwoven single-walled carbon nanotube (SWNT) network, to support large-area graphene nanomesh membrane (GNM) for water desalination (Fig. 5(b)). The robust SWNT network had strong $\pi-\pi$ interactions with the GNM to provide the high mechanical stability. Moreover, the SWNT network could also physically separate the GNM into microsized islands to construct Voronoi cell structures, thus ensuring the structural integrity of the large-area GNM.

Although considerable advancements were made during the past five years, the scale-up of single-layer membranes is still very challenging. The difficulties can be partially relieved by compromising some of the permeance through stacking a few layers (less than 10). ${ }^{12}$ Eliminating the transfer process, the direct and in-situ growth of continuous single-layer porous film on a porous support seems more interesting. Promisingly, the successful efforts in the directed growth of 2D nanosheets, as discussed previously, will offer some insights on the insitu growth of single-layer membranes. 


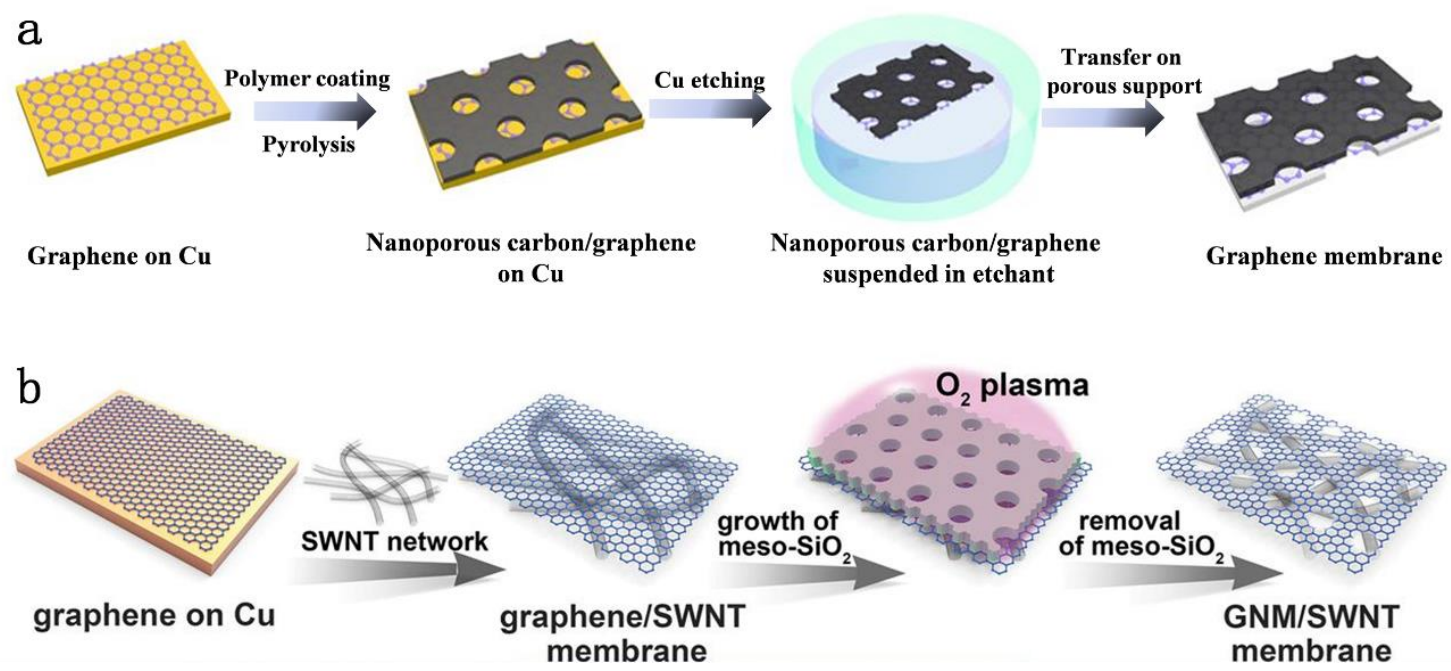

Fig. 5 The transfer of single-layer graphene to a support. (a) A nanoporous-carbon-assisted transfer technique. Reproduced with permission from ref. ${ }^{25}$. Copyright 2018, Springer Nature. (b) An SWNT network-supported transfer technique for large-area GNM. Reproduced with permission from ref. ${ }^{13}$. Copyright 2019, Science Publishing Group.

\subsubsection{Preparation of lamellar membranes}

As a unique feature, 2D nanosheets are solution-processable, which means their suspensions can be easily processed into lamellar structures by using conventional coating or casting methods. ${ }^{11,18}$ Preparing lamellar membranes with nanosheets parallel to the support is easily feasible, and the mass transport distance can be reduced by lowering the membrane thickness or by generating adequate through-plane pores. ${ }^{10} \mathrm{~A}$ perfect lamellar membrane structure should hold parallel and interlocked layers with minimized non-selective edge-to-edge gaps. To achieve this, filtration, coating, layer by layer (LBL) and some combined methods have been applied.

(a) Filtration. Pressure/vacuum filtration (Fig. 6(a)) is one of the most common and effective deposition methods. The vertical downward force, provided by pressure/vacuum filtration, drives the assembly of the $2 \mathrm{D}$ nanosheets into an interlocking layered structure on the substrate. ${ }^{26}$ The membrane thickness can be effectively regulated from nanometer to submicrometer scale by varying the depositing amounts. Moreover, other ions, molecules or nanoparticles can be easily mixed and intercalated into the interlayers, providing additional flexibility on tuning the nanochannel structure. ${ }^{27}$

(b) Coating. Various coating methods have been reported to assemble 2D nanosheets into lamellar membranes, including drop-coating, spay-coating, spin-coating and casting, etc. The success of a uniform coating relies on the smoothness of the substrate, the surface tension of 
the coating solutions, as well as the evaporation process applied. Among the methods, spincoating (Fig. 6(b)) could provide centrifugal and shear forces to control the assembly of the nanosheets, producing a well-interlocked, ordered lamellar structure. ${ }^{10}$ Casting also belongs to the coating strategies which have been widely used in the preparation of polymer membranes. The casting process is continuous and scalable. At present, this technique is also applied to prepare highly ordered lamellar membranes (Fig. 6(c)). ${ }^{11}$ In this process, the shear force, generated by the transverse motion of the doctor blade, promotes the shear alignment of the GO liquid crystal. This method can produce large-area (over $100 \mathrm{~cm}^{2}$ ), uniform and continuous lamellar membranes.

\section{(c) Layer-by-layer (LBL)}

Layer-by-layer (LbL) self-assembly refers to the process of alternately depositing different materials on the substrate surface (Fig. 6(d)). This approach primarily relies on the interactions between the adjacent layers, including electrostatic, hydrogen bonding or even covalent interactions. The LBL method can precisely control the thickness of the selective layer by varying the number of deposition cycles and is beneficial for introducing interlayer stabilizing forces. ${ }^{28}$ Thus, the resultant membranes can remain stable in aqueous or organic media. However, the implementation of this method requires the presence of material interactions and the preparation process is time-consuming. At present, covalent LBL combined with click chemistry etc. has become a new choice for preparing membranes with higher stability and optimized structure.

(d) Combined method. At present, the preparation of the lamellar membrane mostly relies on a single method. A synergetic tuning of the stacking manner in both the horizontal and vertical directions is difficult. Usually, the filtration method can tune the vertical alignment, while spinning or casting methods can tune the horizontal alignment. A combined pressure filtration and spin-coating method was reported to achieve the synergetic regulation of the GO lamellar membranes (Fig. 6(e)). ${ }^{29}$ This process produced a highly ordered lamellar membrane with tailored through-plane and interlayer nanochannels. 
a

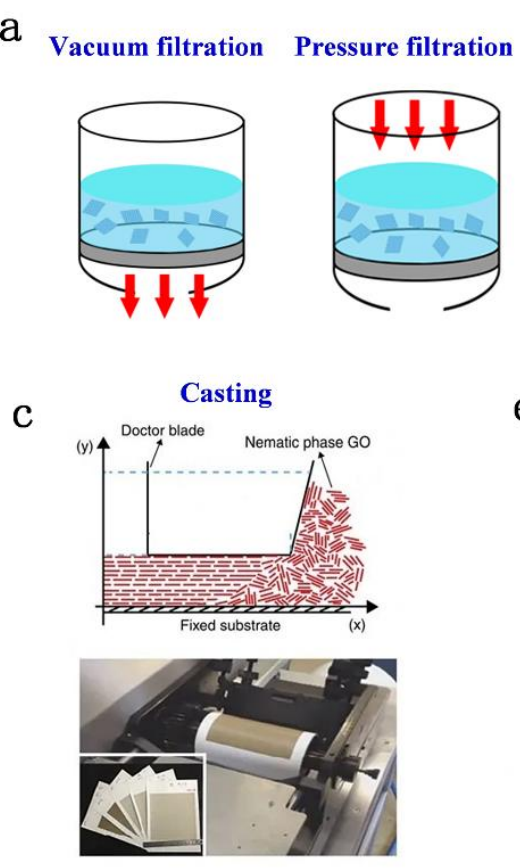

$\mathrm{b}$

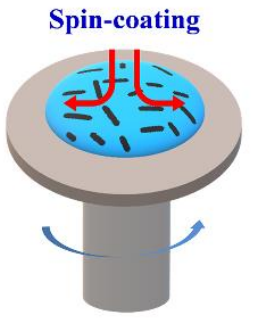

e

Combined method

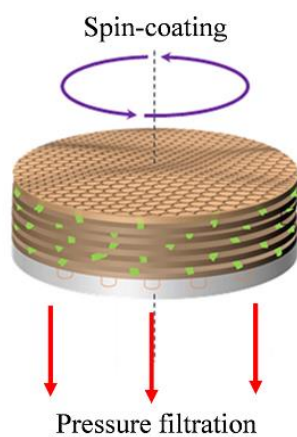

d Layer-by-layer
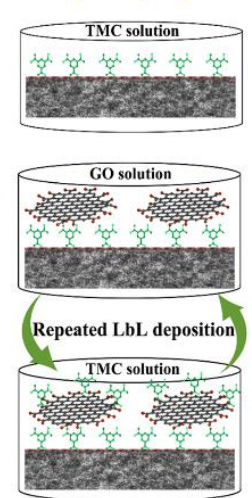

Fig. 6 Representative preparation methods of lamellar membranes. (a) Pressure/Vacuum filtration. (b) Spin-coating. (c) Casting with shear alignment. Reproduced with permission from ref. ${ }^{11}$. Copyright 2018, Springer Nature. (d) Layer by Layer. Reproduced with permission from ref. ${ }^{28}$. Copyright 2013, American Chemical Society. (e) Combined method. Reproduced with permission from ref. ${ }^{29}$. Copyright 2016, American Chemical Society.

The scalability of membrane preparation methods is essential to implement practical applications. Coating and casting methods are scalable and industrially adaptable methods for thin lamellar flat sheet membranes. In addition, attaching 2D nanosheets to hollow fibers by filtration methods could facilitate the translation from the laboratory scale to the industrial scale. ${ }^{30}$ Note that, all these methods are fundamentally based on a uniform suspension of nanosheets, accordingly, selecting appropriate solvents to match the hydrophilicity of the nanosheets or implementing necessary modifications to improve the dispersion are important pre-steps. $^{18}$

\section{Nanochannel architecture and microenvironment regulation}

Nanochannel is the core of 2DNCMs, which connects the membrane preparation and the separation process. In 2DNCMs, the architecture and microenvironments of nanochannels determine the ultimate separation performance of the membranes. The fine-tuning of the channels is therefore essential. In the following section, the regulation strategies of different channels and the effects of regulation on molecular and ionic separation are discussed. 


\subsection{Nanochannels based on nonporous 2D nanosheets}

The lamellar membrane stacked by nonporous 2D nanosheets is primarily separated by interlayer nanochannels. The channel size and chemical functionalities play decisive roles in the separation process. ${ }^{10}$ The formation mechanism of nanochannels can be attributed to interlayer interactions, including non-covalent and covalent interactions. Therefore, as illustrated in Fig.7, the nanochannels could be regulated by adjusting these interactions. The non-covalent interactions are further divided into internal force, external force, and physical intercalation, classified by the type of forces to drive the formation of nanochannel. Note that, we separately discuss internal forces and external forces. Internal forces refer to intrinsic interactions derived from adjacent monolayers, including hydrogen bonding, hydrophobic effects, van der Waals and $\pi-\pi$ interactions. The internal forces play a critical role in holding the multiple layers together. In contrast, the external forces (centrifugal force, pressure, etc.) applied during the channel formation or operation are essential to provide a favourable microenvironment.

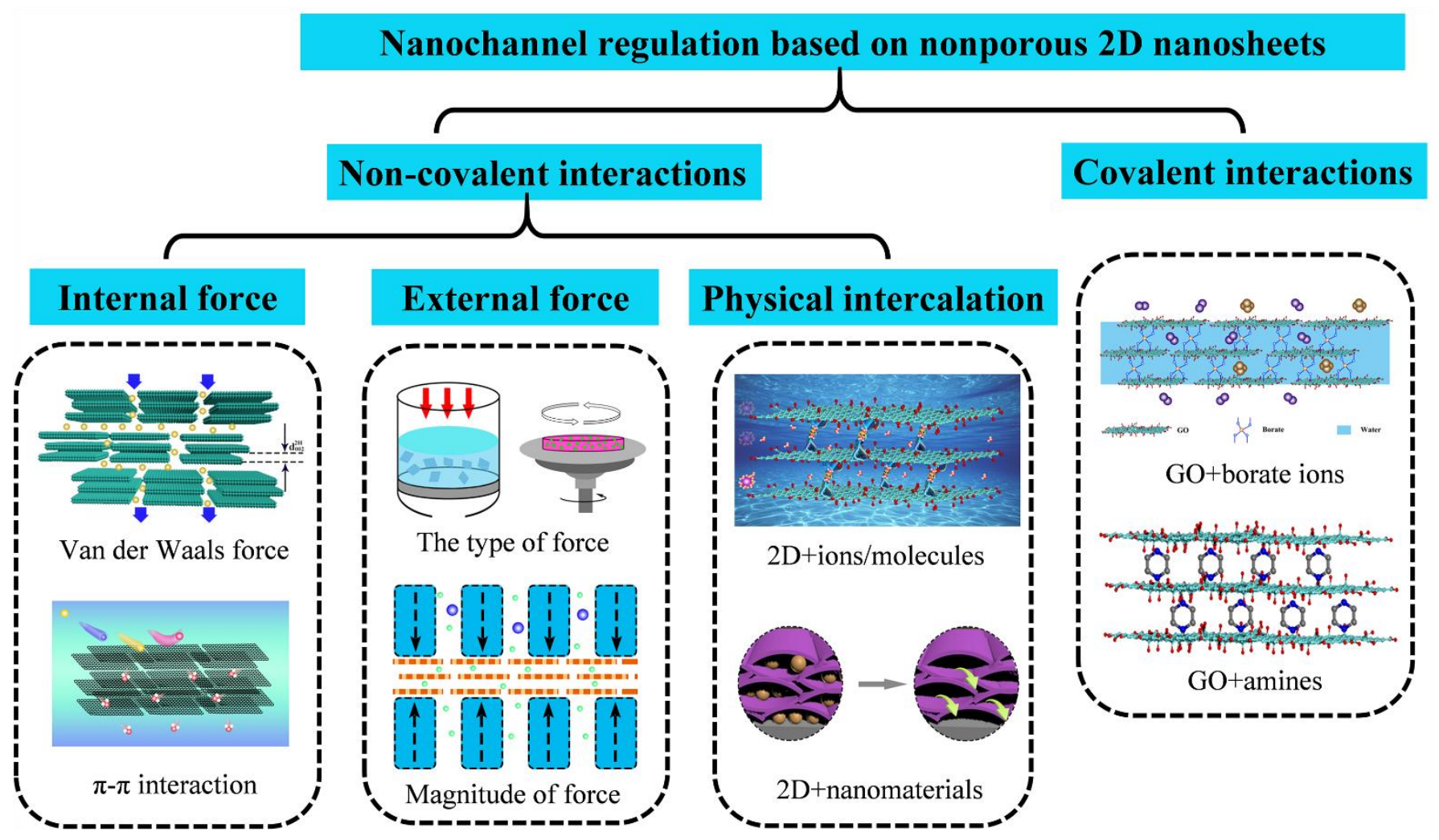

Fig. 7 Interlayer nanochannel regulation based on different forces. Van der Waals force, reproduced with permission from ref. ${ }^{20}$. Copyright 2016, Royal Society of Chemistry. Magnitude of force, reproduced with permission from ref. ${ }^{31}$. Copyright 2018, American Chemical Society. $2 \mathrm{D}+$ ions/molecules $\left(\mathrm{GO}+\mathrm{K}^{+}\right)$, reproduced with permission from ref. ${ }^{32}$. Copyright 2017 , Springer 
Nature. 2D+nanomaterials ( $\mathrm{MXene}+\mathrm{Fe}_{3} \mathrm{O}_{4}$ nanoparticles), reproduced with permission from ref. ${ }^{27}$. Copyright 2017, John Wiley and Sons.

\subsubsection{Architecture and microenvironment regulation based on non-covalent interactions}

\section{Regulation based on internal forces}

In lamellar membranes, the interlayer interactions such as van der Waals, $\pi-\pi$ stacking etc, are the internal forces to hold the adjacent layers. Thus, the aperture of interlayer nanochannels can be regulated by varying the intensity of interlayer forces through thermal or chemical treatment. The treatment process can be conducted before or after membrane formation.

From the material aspect, a uniform covering of functional groups on the nanosheets will produce uniform interlayer forces to bring well-ordered structure. Thus, compared with GO that has randomly distributed oxygen groups, MXenes decorated with abundant surface-terminating groups can easily form regular lamellar structures. The regular free spacings generated by interlayer van der Waals interactions could perform as efficient size-sieving nanochannels for $\mathrm{H}_{2}$ separation. ${ }^{33}$

The oxygenated groups on GO prevent the interlayers from restacking by $\pi-\pi$ interactions. ${ }^{10}$ Therefore, enhancing the interlayer $\pi-\pi$ interactions via thermal annealing or chemical reduction could produce narrowed nanochannel size and more stable channel structures. ${ }^{10}$ In contrast, the electrochemical reduction method is also reported to selectively remove the oxygencontaining groups on GO, leading to a more precise control over the size of nanochannels. ${ }^{34}$ Within 20 seconds, there is a linear relationship between the GO reduction degree and the electrophoresis-applied time (Fig. 8(a)). The resultant reduced GO membranes featured a strict molecular sieving characteristic between C2 (ethane and ethene) and C3 (propane and propene) hydrocarbons and nearly complete rejections for the smallest alcohol and ions.

The van der Waals forces of $\mathrm{MoS}_{2}$ nanosheets can also be adjusted by thermal annealing, but the channel-tuning mechanism is different from that of GO. The thermal annealing process can induce a phase transition of $\mathrm{MoS}_{2}$ from $1 \mathrm{~T}$ to $2 \mathrm{H}$, which enhances the interlayer van der Waals interactions to form few-layer $\mathrm{MoS}_{2}$ bundles. $^{20}$ The resultant membrane showed tightened interlayer channels but widened inter-bundle channels, through which the gases predominantly transport (Fig. 8(b)). Surprisingly, the simple phase transition could increase the $\mathrm{H}_{2}$ permeability of $2 \mathrm{H} \mathrm{MoS}_{2}$ membrane by $30 \%$ compared with the as-prepared $1 \mathrm{~T} \mathrm{MoS}_{2}$ membrane. 

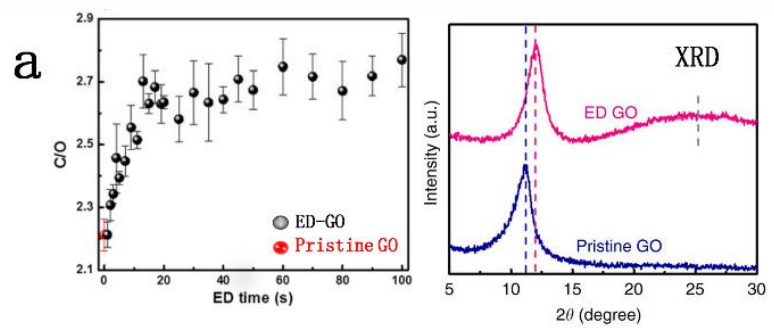

C

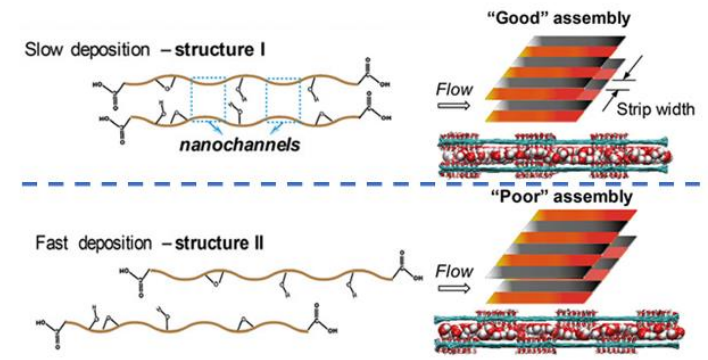

b

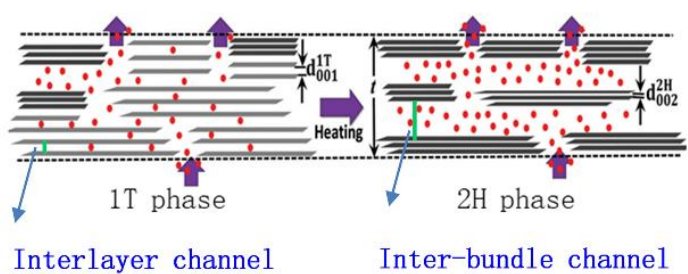

d

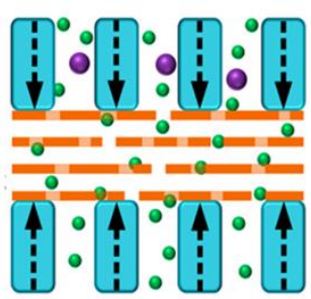

Fig. 8 Architecture and microenvironment regulation of lamellar membranes based on the internal and external forces. (a) $\mathrm{C} / \mathrm{O}$ ratio of the $\mathrm{GO}$ submitted to the different time duration of ED (left), and the corresponding XRD patterns. Reproduced with permission from ref. ${ }^{34}$.

Copyright 2017, Springer Nature. (b) Effect of heating on the conversion of the 1T MoS 2 (grey sheets) to the $2 \mathrm{H}$ phase (black sheets) and the molecular transport pathways. Reproduced with permission from ref. ${ }^{20}$. Copyright 2016, Royal Society of Chemistry. (c) Interlayer structures of GO membranes prepared by slow and fast deposition rates (left), and sketch of water transport through nanochannels (right). Reproduced with permission from ref. ${ }^{35}$. Copyright 2017, American Chemical Society. (d) Interlayer channel size of the GO membrane under different pressures. Reproduced with permission from ref. ${ }^{31}$. Copyright 2018, American Chemical Society.

\section{Regulation based on external forces}

During the deposition of the nanosheets, the external forces (pressure, shear force, etc.) can be adjusted to align the nanochannels, enabling the effective control of their regularities. GO membranes are typical examples. GO membranes prepared by pressure filtration, vacuum filtration or evaporation show ordered, partially ordered and random nanochannels characteristics respectively. ${ }^{26}$ Generally, both evaporation and drop-coating methods rely on the slow evaporation of solvents to form a lamellar structure, which is usually less ordered. ${ }^{17}$ By applying the shear force from spin-coating, a better-interlocked structure can be achieved. The combined method, by simultaneously exerting pressure in the vertical direction, shear force and centrifugal force in the horizontal direction, can produce a highly ordered structure, better than any single method (pressure filtration, spin-coating). ${ }^{29}$ As a unique case, when GO liquid 
crystals are cast under a transverse shear-force, the nanosheets are arranged in a nearly horizontal arrangement. ${ }^{11}$ This method allows the highest degree of ordering among the current lamellar membrane preparation methods. Based on the above analysis, the estimated ordering of the lamellar membrane produced by different methods varies from low to high: drop coating (evaporation, LBL), vacuum filtration, pressure filtration (spin-coating), combined method and shear alignment of liquid crystals (Fig. 9).

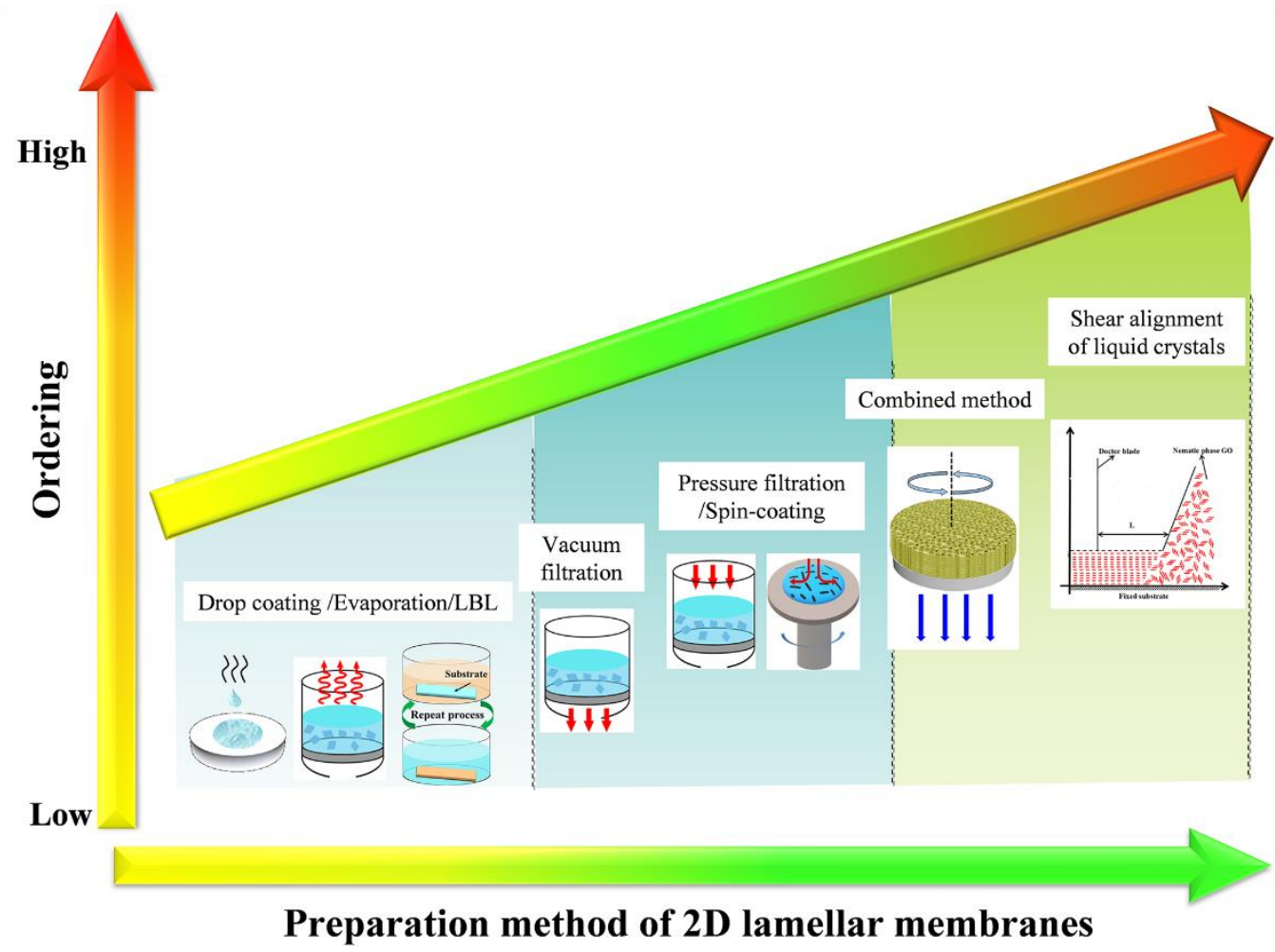

Fig. 9 Different preparation methods and the corresponding channel ordering of 2D lamellar membranes. The combined method, reproduced with permission from ref. ${ }^{29}$. Copyright 2016 , American Chemical Society. The shear alignment of liquid crystals, reproduced with permission from ref. ${ }^{11}$. Copyright 2018, Springer Nature.

Besides the type of forces, their magnitude gives higher tuning precision on the stacking manner, especially for nanosheets with distributed functionalities. Geim's group ${ }^{36}$ first propose the low-friction, rapid transport of water in graphene nanocapillaries of GO membranes. To amplify this mechanism, the oxygen groups should not present in the nonoxidized domains from the adjacent layers. A slow deposition process is therefore necessary, which guarantees the sufficient stretching and the hydrophilicity matching of adjacent nanosheets. As reported, the channel size of the GO membrane prepared by a slow deposition process was $3.5 \AA$, less than that prepared by a fast deposition process $(3.8 \AA) .{ }^{35}$ Meanwhile, the slow deposition leads to the 
oxidized subdomains facing another oxidized subdomain $(\mathrm{O}-\mathrm{O})$, pristine subdomains facing another pristine subdomain (P-P) in GO nanosheets (Fig. 8(c)). The P-P domains constitute more effective mass transport pathways, affording the well-distributed hydrophilic-hydrophobic nanodomains and favouring the water transport. This study elucidates the effect of GO interlamellar microstructure on the transport of water molecules, which is also applicable to promote the transport of other polar molecules, like $\mathrm{CO}_{2}{ }^{37}$

The interlayer nanochannel size can be also be possibly regulated during membrane operations. The extra external forces, like a high operating pressure can compact the interlayer channels and restrain the swelling of lamellar membranes. When the external pressure increased from 0 to $10 \mathrm{MPa}$, the corresponding channel size of $\mathrm{GO}$ membrane decreased from $8.3 \AA$ to $4.5 \AA$ (Fig. 8(d)). ${ }^{31}$ The pressure regulation strategy triggered an attempt to regulate 2DNCMs in the separation process, achieved high $\mathrm{KCl}, \mathrm{NaCl}, \mathrm{CaCl}_{2}$ rejections. This type of regulation requires a fixed pressure range, which might not be suitable for other pressures. However, it would help to explain the performance variation of lamellar membranes operated at different pressures. ${ }^{37}$

In brief, the regulation of internal forces and external forces mainly relies on the intrinsic properties of the $2 \mathrm{D}$ nanosheets. The regulation precision can be satisfactory, but the regulation range of channel size is still limited. In most cases, a uniform interlayer spacing is required to guarantee the size sieving. However, when the interlayers are too tightly bonded, the presence of disordering may render more permeation pathways to achieve a higher permeance. ${ }^{17}$

\section{Regulation based on interlayer physical intercalation}

Physical or chemical intercalation of ions, molecules or nanomaterials into the layers seems a simple but effective method to regulate the interlayer nanochannels. The nanochannel size can be determined by the size of spacers, resulting in a wide range of control. ${ }^{38}$

The small size of molecules or ions offers an opportunity to regulate the interlayer channels suitable for gas or liquid separations. Divalent cations are well-known to generate strong chelate bonds with GO. The mechanical stiffness can be enhanced by $200 \%{ }^{39}$ Recently, noncovalent yet strong cation- $\pi$ interactions between hydrated cations and the aromatic rings from GO nanosheets was firstly reported. The nanochannel size of GO membranes was regulated with a precision of angstrom, from $11.4 \AA$ to $13.6 \AA$, using $\mathrm{K}^{+}, \mathrm{Na}^{+}, \mathrm{Ca}^{2+}, \mathrm{Li}^{+}$or $\mathrm{Mg}^{2+}$ ions (Fig. 10 (a)). ${ }^{32}$ Surprisingly, the nanochannel size controlled by one type of cation (e.g. $\mathrm{K}^{+}$) can efficiently and selectively exclude other cations that have larger hydrated volumes. The stable membrane can be applied for precise ion and molecular sieving in aqueous solution. 
Nanomaterials with larger sizes than ions and molecules can afford wider channel openings. The dimensions of nanomaterials span from OD to 3D. Recently, a MXene membrane with arched-structure was prepared by intercalating nanoparticles (Figure 10(b)). ${ }^{27}$ The negatively charged MXene nanosheets were tightly combined with the assistance of positively charged OD $\mathrm{Fe}(\mathrm{OH})_{3}$ nanoparticles $(4-5 \mathrm{~nm})$ to form an arch-bridge structure. Subsequently, after etching the $\mathrm{Fe}(\mathrm{OH})_{3}$ nanoparticles with a hydrochloric acid solution, the arched-structure was preserved and formed expanded nanochannels. The MXene membranes with expanded nanochannels facilitated water molecules rapid transport and exhibited high water permeance (more than 1000 $\mathrm{L} \mathrm{m}^{-2} \mathrm{~h}^{-1}$ bar $\left.^{-1}\right)$. The combination of different types of 2D material, has also received increasing attention. An appropriate combination will balance the trade-off between permeance and rejection, as well as between separation performance and mechanical stability.

In summary, non-covalent nanochannel regulation mainly depends on the interlayer forces, with facile and controllable characteristics, and with a precision of angstrom. Moreover, the combination of ions, molecules or various dimensional nanomaterials with 2D nanosheets provides more possibility for the construction of hierarchical channels. In this case, the amount and their distribution of the intercalators should be carefully designed, since the intercalators may also possibly hinder the transport of penetrants.
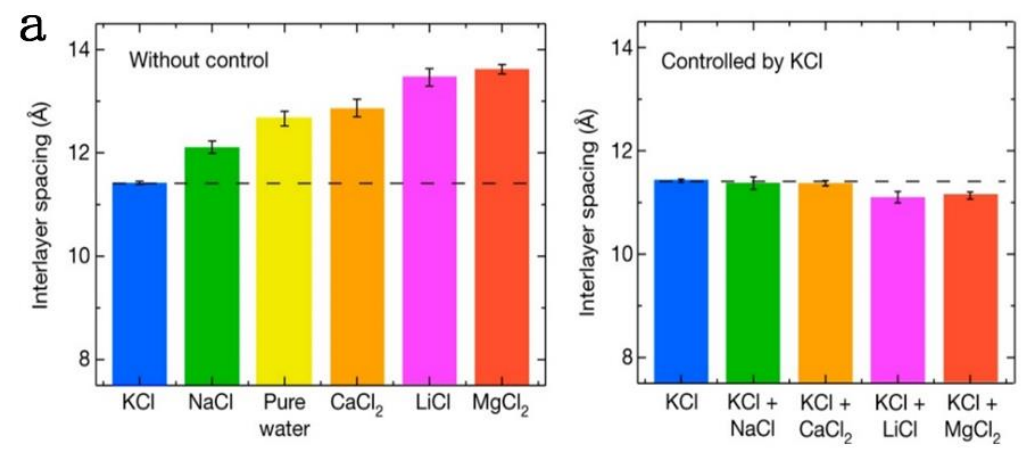

b
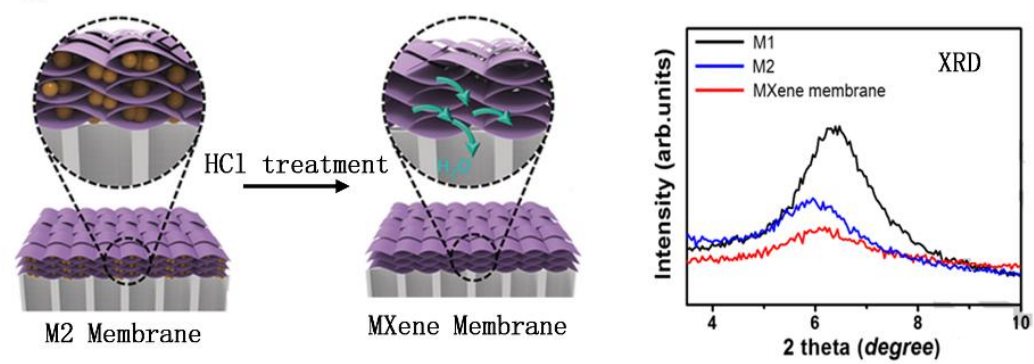

Fig. 10 Structural regulation of lamellar membranes based on the physical intercalation. (a) Interlayer spacings of GO membranes that were immersed in various solutions (left). Interlayer spacings of $\mathrm{GO}$ membranes that were soaked in $\mathrm{KCl}$ solution, followed by immersion in various 
salt solutions (right). Reproduced with permission from ref. ${ }^{32}$. Copyright 2017, Springer Nature. (b) A MXene membrane with arched-structure. The membrane preparation method (left). XRD spectrum to show the enlarged interlayer spacings of MXene membrane after intercalation (right). Reproduced with permission from ref. ${ }^{27}$. Copyright 2017, John Wiley and Sons.

\subsubsection{Architecture and microenvironment regulation based on covalent interactions}

For nanosheets that can be covalently bonded, chemical intercalations by cross-linking are the common strategy to strengthen the interlayer forces and to improve the mechanical stability of the membranes. The cross-linkers encompasses ions, small molecules or macromolecules.

Considering the rich functional groups (epoxide, carboxyl, carbonyl, and hydroxyl), GO is the mostly explored nanosheet for chemical intercalation. Our recent work demonstrated that a borate ion cross-linked GO nanosheets possessed controllable nanochannels (Fig. 11(a)). ${ }^{40} \mathrm{~A}$ dehydration-condensation reaction occurs between borate ions and $\mathrm{C}-\mathrm{OH}$, forming a strong $\mathrm{C}$ O-B and B-O-B covalent network. By simply increasing the annealing temperature, the channel size decreased from $4.2 \AA$ to $3.3 \AA$, which is exactly between the kinetic diameters of $\mathrm{CO}_{2}$ and $\mathrm{N}_{2} / \mathrm{CH}_{4}$, and as a result, the membrane exhibited molecular sieving properties. Moreover, the borate in the GO interlayer can greatly facilitate the transport of $\mathrm{CO}_{2}$ via the carbonic anhydrase mechanism. Through the synergy of physical and chemical microenvironments in the channels, a high-performance $\mathrm{CO}_{2}$ separation membrane is constructed. This borate cross-linking strategy inspires the rational design of MXene membranes with tuneable $\mathrm{H}_{2}$ or $\mathrm{CO}_{2}$-selective gas transport channels. ${ }^{41}$

Diamines are the popular cross-linkers for GO, through a nucleophilic substitution reaction between amine and epoxy. The membrane applications vary from gas separation, water purification and organic solvent nanofiltrations. Due to the rich diversity of diamines, the interlayer spacings can be facilely regulated by varying the chain length of diamines. ${ }^{37,} 42$ Recently, another kind of diamine, piperazine, has also been proved effective for GO crosslinking (Fig. 11(b))..$^{30}$ Through a two-step vacuum filtration, ultrathin GO membranes were uniformly deposited on the inner surface of a hollow fiber. After a thermal treatment, piperazines were chemically intercalated between the GO layers. The partially cross-linked, brush-like piperazines functioned as semi-mobile carriers, creating a chemical microenvironment favouring for $\mathrm{CO}_{2}$ transport. The membrane exhibited extremely high permeance and superior selectivity for $\mathrm{CO}_{2} / \mathrm{N}_{2}$ separation. Considering the hollow fiber configuration, this membrane should be potentially used for flue gas treatment. 

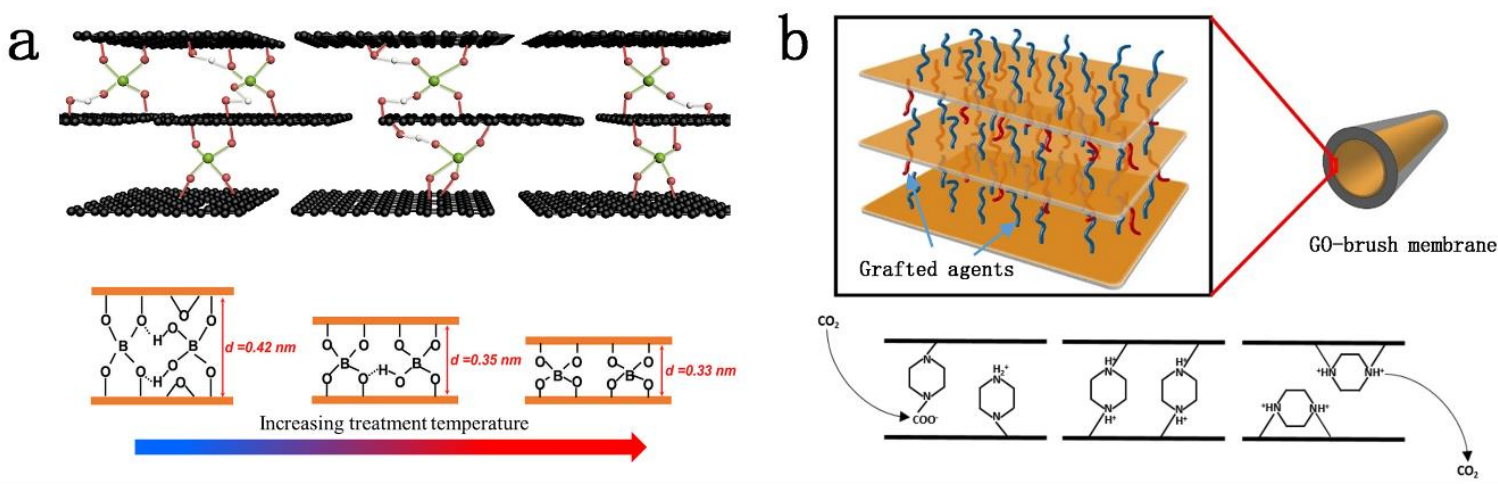

Fig. 11 Structural regulation of lamellar membranes based on covalent interactions. (a) Schematic of GO crosslinked with borate ions and the nanochannel size of GO-B membranes by heating treatment. Reproduced with permission from ref. ${ }^{40}$. Copyright 2016, Royal Society of Chemistry. (b) The structure diagram of the GO-based hollow fiber membranes (top). The $\mathrm{CO}_{2}$ transport inside the GO layers (down). Reproduced with permission from ref. ${ }^{30}$. Copyright 2017, Springer Nature.

The covalent intercalation requires the presence of specific functional groups on the nanosheets, and the strong covalent interactions can guarantee the structural integrity to avoid swelling in liquid separations. ${ }^{19,} 42$ The intercalated molecules can not only crosslink 2D nanosheets to tune the channel size (physical microenvironment), but also introduce penetrantphilic groups (chemical microenvironment) into the nanochannels, for transporting targeted molecules. As an adverse effect, the introduction of covalent intercalators may cause sever aggregation of nanosheets in the casting solutions. The aggregation can be partially retarded by slowing down the reaction via decreasing reaction temperature or crosslinker concentrations.

\subsection{Nanochannels based on intrinsically porous 2D nanosheets}

Besides the nanochannels formed by the interlayer spaces, when using nanosheets with intrinsic porosity, greater opportunities for building selective transport paths are created. This versatility can be provided by zeolite, MOFs and COFs nanosheets. Most zeolites have pore sizes in the range of $2-10 \AA$, close to the molecular sizes of most gases. While, the pore size distribution of MOFs is quite broad, from 3 to $100 \AA$, according to the size of the organic linkers. MOFs membranes can be suitable for gas or liquid separations. For COFs, the reported pore size ranges from 8-50 $\AA$, which is larger than that of industry-relevant gas molecules. Intrinsically porous 2D nanosheets are usually brittle because of the high crystallinity. Especially when they are processed into single-layer membranes, the structural defects are inevitable. ${ }^{22}$ 
Adopting appropriate sealing methods, e.g., secondary growth, or covering with polymeric or inorganic materials, is the pre-step before channel regulation. ${ }^{21}$

The nanochannel regulation is mainly focused on the pre-design of nods and linkers of the starting porous 2D nanosheets. ${ }^{17}$ Based on the molecular size of the penetrants, the building units with proper sizes can be selected. Herein, we highlight a few pioneering works that the tuneable channel can affect the membrane separation performances. Recently, COFs are a rising star in membrane science. The robust covalent bonds in COFs bring about higher chemical stability and higher processability compared with MOFs or zeolites. The Banerjee's group $^{43}$ realized the interfacial synthesis of COF films under ambient conditions by adopting a salt mediated method. They used 1,3,5-triformylphloroglucinol (Tp) as a fixed building unit. By changing the length and geometry (linear or triangle) of the other amine organic linker, four kinds of COFs films were synthesized with pore sizes ranging from 14 to $26 \AA$ (Fig. 12 (a)). The corresponding COFs membranes exhibited a tuneable permeation and rejection performance in organic solvent nanofiltration. Keeping the same backbone of building units ( $\mathrm{C}_{3}$-symmetric hexamine and $\mathrm{C}_{2}$-symmetric tetraone), a pre-modification approach could also be applied to synthesize 2D COFs nanosheets. Through a combination of different functional groups, 25 kinds of COFs could be produced in gram amounts. (Fig. 12(b)). ${ }^{15}$ The highly ordered and modifiable COFs nanosheets were composed of a very stable aromatic backbone and functionalized pores. The pore size is adjustable in the range of 19-28 $\AA$. By introducing the hydrophilic ionizable carboxylate groups in $2.8 \mathrm{~nm}$ diameter pores, the water flux was greatly enhanced in the COF 9 membrane. The COFs 8 and 9 best illustrated the formation of positively or negatively charged pores and were applicable to different charge rejections. This work demonstrated that both the pore sizes and chemical functionalities can be simultaneously tuned to achieve enhanced separation performances.

Instead of tuning the intrinsic pore structure, tuning the assembly architecture of porous nanosheets is easier to operate. As mentioned earlier, the pore size of the COF nanosheets is usually larger than most gas molecules. The introduction of functional groups in COFs to narrow the pore size is a traditional way forward to enhance the selectivity. ${ }^{15}$ On the other hand, for lamellar membranes made from porous 2D nanosheets, the misalignment of pores along the vertical direction is undesired, since the mass transport will take place in a tortuous way. ${ }^{17}$ However, the misalignment may also bring potential benefits. Recently, an impressive COF membrane for gas separation has been reported by Caro and co-workers. ${ }^{44}$ They prepared COF-COF bilayer composite membranes by successively regulating the growth of imine-based 
COF-LZU1 and azine-based ACOF-1 layers via a temperature-swing solvothermal approach. The COF-LZU1 and ACOF-1 have both a hexagonal pore structure with an aperture size of $\sim 18$ and $\sim 9.4 \AA$. The formed interlaced pores of the COF-LZU1-ACOF-1 membrane are in the appropriate size range of the kinetic diameter of gas molecules to enable size sieving (Fig. 12(c)). The as-prepared bilayer membranes lead to a much better separation selectivity for $\mathrm{H}_{2}$ over $\mathrm{CO}_{2}, \mathrm{~N}_{2}$, and $\mathrm{CH}_{4}$ due to the formation of small interlaced pores (3-5 $\AA$ ) at the interface, opening a new route to regulate the pore size of COFs.

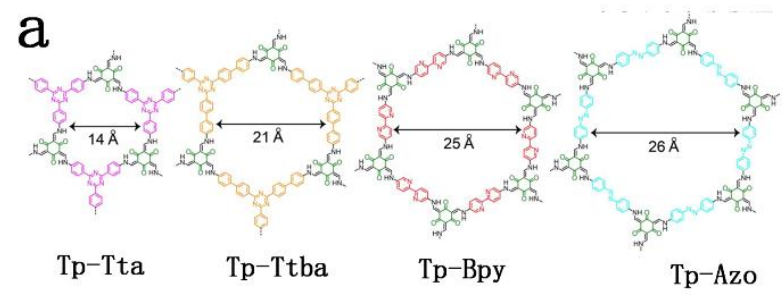

b
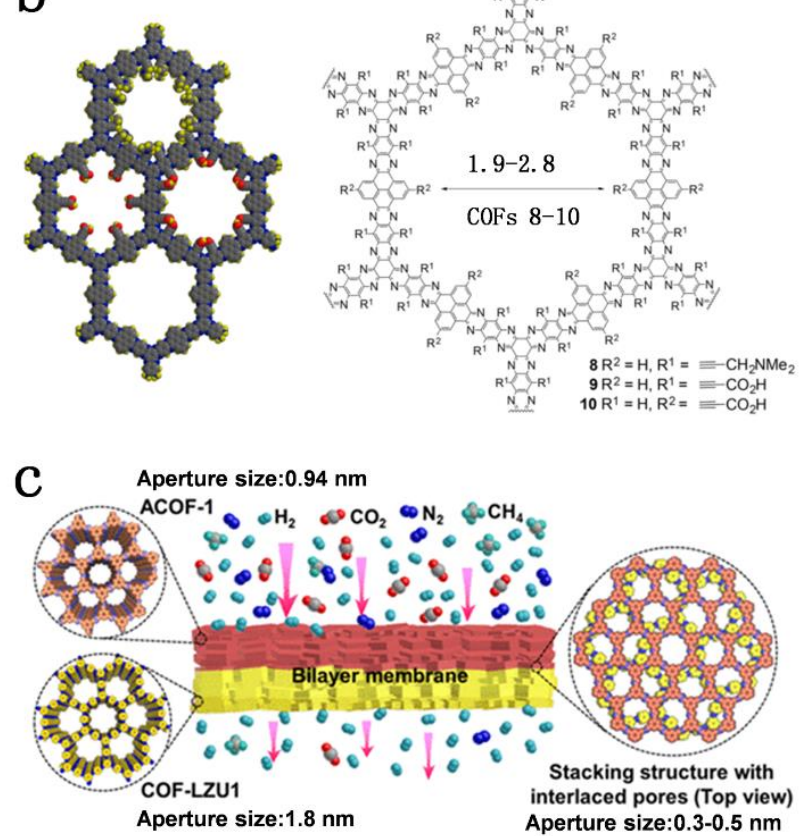

Fig. 12 Nanochannel size control and functionalization in porous 2D nanosheets. (a) Pore size tuning of a COF material via different linker lengths. Reproduced with permission from ref. ${ }^{43}$. Copyright (2017) American Chemical Society. (b) Pre-modification of the backbone of building units to achieve COF nanosheets with different pore sizes and functionalities. Left: space-filling model, illustrating the individual pore structure of COFs 8-10. Right: the corresponding chemical functionalities. Reproduced with permission from ref. ${ }^{15}$. Copyright 2018, American Chemical Society. (c) A COF-COF bilayer structure to produce interlaced pores effective for gas 
separation. Reproduced with permission from ref. ${ }^{44}$. Copyright 2018, American Chemical Society.

To sum up, owing to the rich chemistries involved in the 2D porous nanosheets synthesis, both the channel size and chemical functionality can be facilely manipulated. However, most of the researches still focus on material exploitation, only a small portion has been translated to membranes for target separations. Moreover, the rich combination of different nanosheets, e.g., MOFs-COFs, COFs-COFs, GO-MOFs, has highlighted some promising directions to the channel size and functionality regulation.

\subsection{Nanochannels based on perforated 2D nanosheets}

Generating of nanopores in the 2D nanosheets stands for another important channel regulation strategy, through which simulation studies have been conducted on graphene with various modifications, graphyne, $\mathrm{h}-\mathrm{BN}, \mathrm{MoS}_{2}$, etc. ${ }^{5}$ The experimental success has mostly seen in graphene material, due to its superior mechanical strength and the well-explored pore generating techniques. The numerous perforation approaches include physical methods (focused electron beam (FEB) irradiation, focused ion beam (FIB) irradiation, ion bombardment, etc.) and chemical methods (chemical oxidative etching, plasma, ultraviolet (UV) light etching, etc.).

The FEB method applies high energy (hundreds of keV) electron beam to effectively etch carbon atoms and induce structure rearrangements, thus forming pores on graphene. By controlling the parameters of FEB (voltage, time, beam dose), the pores diameter can be tuned from micron to nano meter scale. In the FIB process, graphene is subjected to the irradiation of ions beam to fabricate nanochannels of 5-100 nm. Generally, the FIB and FEB irradiation can make a well-defined channel size, with a resolution of $1 \mathrm{~nm}$, but it is limited by a low pore density and relatively small areas. ${ }^{45}$ Chemical methods like UV-induced oxidative etching and ozone-etching can introduce $\mathrm{sp}^{3}$-hybridized sites or vacancy defects by carbon-etching on graphene. In these processes, the functional groups (carbonyl, hydroxyl, etc) formed by oxidation treatment migrate, rearrange, and finally desorb $\mathrm{CO}$ or $\mathrm{CO}_{2}$ forming channels with a precision of angstrom. ${ }^{46}$ The pioneering work by Bunch et al. ${ }^{9}$ revealed the gas-selective nanopores can be achieved by UV-induced oxidative etching on micrometer-sized graphene membranes. A breakthrough in optimizing the pore density and pore size distribution was achieved by Celebi et al. ${ }^{12}$ They applied $\mathrm{Ga}$ and He-based FIB to generate a few million 
controlled pores ranging from $7.6 \mathrm{~nm}$ to $1 \mu \mathrm{m}$, in a $4 \mu \mathrm{m}$ double-layer graphene (Fig. 13 (a)). Low exposure doses enabled fast and precise drilling, leading to well-defined pore diameter distributions. The graphene membranes with $7.6 \mathrm{~nm}$ nanopores exhibited Knudsen diffusion selectivity for $\mathrm{H}_{2} / \mathrm{CO}_{2}$ (4.69) and ultrahigh $\mathrm{H}_{2}$ permeance values $\left(\sim 10^{-2} \mathrm{~mol} \mathrm{~m}^{-2} \mathrm{~s}^{-1} \mathrm{~Pa}^{-1}\right)$, which was 3 5 orders of magnitude superior to other highly-permeable polymeric, inorganic and GO membranes, ${ }^{12}$ highlighting the ultimate permeation these 2D membranes can provide.

Despite significant progress, obtaining high-precision and high-density nanochannels on graphene membrane by a single perforation method still faces many challenges. It was reported that the coupling of two perforation technologies could be effective to fabricate single-layer porous membranes. Examples are a combination of oxygen plasma etching or ion bombardment followed by chemical oxidative etching, generating porous graphene with tuneable pore size with a high pore density $\left(2.1 \times 10^{12} \mathrm{~cm}^{-2}\right)$, and size resolution down to subnanometer used in nanofiltration process. ${ }^{47}$ However, a more precise regulation, down to the angstrom level, is still challenging. Very recently, a synergistic, partially decoupled defect nucleation and pore expansion strategy was proposed. ${ }^{45}$ Firstly, by a 1-s $\mathrm{O}_{2}$ plasma, a highdensity $\left(\sim 10^{12} \mathrm{~cm}^{-2}\right)$ of $\mathrm{sp}^{3}$ defects (oxygen-functionalized sites) or vacancy defects (smaller than $2.9 \AA$ ) was nucleated. Subsequently, the activated nuclei could be expanded in a controllable manner by tuning the dynamic (regulating temperature) and the time of the reaction between $\mathrm{O}_{3}$ and graphene defects $\left(\mathrm{sp}^{3} \mathrm{C}\right)$, resulting in the pores with a precision of angstrom. Through the synergistic, partially decoupled strategy, an atom-thick graphene membrane with an angstrom precision and a high pore density (up to $2.1 \times 10^{12} \mathrm{~cm}^{-2}$ ) was achieved. The angstrom precision nanopores, limiting the percentage of $\mathrm{CH}_{4}$ permeating to 13-22 parts per million, control the gas diffusion process of membrane and exhibit size-sieving properties. As a result, the nanoporous atomically thin membrane exhibits a record-high gas mixture separation performance with high $\mathrm{H}_{2}$ permeance (6045 GPU) and appropriate $\mathrm{H}_{2} / \mathrm{CH}_{4}$ selectivity (25.1) (Fig. 13(b)). This highly precision pore (sieving-resolution of $1 \AA$ ) etching strategy will accelerate the development of atom-thick 2D energy-efficient membranes. 

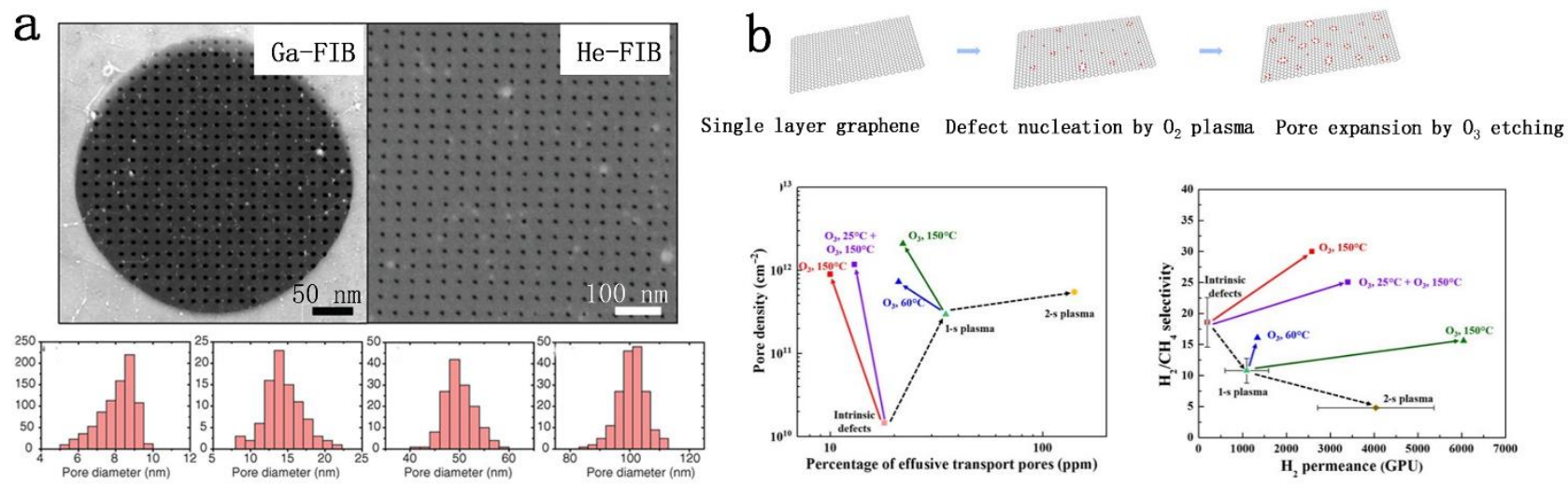

Fig. 13 Representative nanopores graphene fabricated by different perforation methods. (a) SEM images of Ga and He-based FIB-drilled graphene, and the pore size distributions (down). Reproduced with permission from ref. ${ }^{12}$. Copyright 2014, Science Publishing Group. (b) Schematic of the combined strategy to perforate graphene (top), and the evolution of graphene nanopores (down left) and its impact on the separation performance (down right). Reproduced with permission from ref. ${ }^{45}$. Copyright 2019, Science Publishing Group.

In brief, the perforation approaches have demonstrated more precise control over pore size and density, verifying the various simulation results. The resultant single-layer membranes, rigorously tested in exquisite set-ups, are mostly applied in liquid separations. Perforating angstrom-precision pores suitable for small molecular separation and expanding the membrane size to even centimeter scale, remains challenging and far from real applications.

\section{Applications}

A finely tuned nanochannel would create favourable microenvironments to enhance molecular and ionic separation performances. The nanochannel size range of $3-20 \AA$ is especially attractive for the precise sieving of gas, liquid or ions, as evidenced by the fact that some of the 2DNCMs exhibit superior separation performance far beyond the existing polymeric membranes. In this section, some high-performance 2DNCMs used for gas molecular separation, liquid molecular separation, and ionic separation are briefly covered.

\subsection{Gas molecular separations}

The separation of gas mixtures is essential in industrial processes such as hydrogen purification, $\mathrm{CO}_{2}$ capture, and air separation. Compared with other separation systems, gas separation is more defect-sensitive, which raises a higher requirement for the channel regulation. Gas separation through the interlayer channels in GO membranes was first reported by Park's group. ${ }^{10}$ The high humidity environment promoted the $\mathrm{CO}_{2}$-philic permeation. The 
intercalation of extra species into 2D nanosheets is currently a growing research topic to enhance the separation performances. It has been demonstrated that the borate cross-linked GO nanosheets simultaneously regulated the physical and chemical microenvironments of the nanochannels. ${ }^{40}$ And the $\mathrm{CO}_{2}$ permeance and $\mathrm{CO}_{2} / \mathrm{CH}_{4}$ selectivity of ultrathin $(<10 \mathrm{~nm}) \mathrm{GO}$ membrane were significantly improved from 105 GPU, 16 to 650 GPU, 75. Interestingly, the presence of borate ions plays a decisive role in improving performance. Recently, an even higher performance was achieved by introducing $\mathrm{CO}_{2}$-philic piperazine as a cross-linker and semi-mobile carrier into GO interlayer. ${ }^{30}$ The membrane exhibited excellent separation performance under humidified conditions with high $\mathrm{CO}_{2}$ permeance (1020 GPU) and superior $\mathrm{CO}_{2} / \mathrm{N}_{2}$ selectivity (680). The channel size of the membrane was reported as $6 \AA$, which was larger than the kinetic diameters of rejected molecules. In their case, the piperazine with high $\mathrm{CO}_{2}$ affinity has a major contribution to the superior selectivity of the membrane.

MXene is an emerging nonporous 2D nanosheet, which has abundant surface-terminating groups. Ding et al. $^{33}$ assembled MXene into a highly ordered lamellar membrane with a nanochannel around $3.5 \AA$, which could serve as a molecular sieve channel. The MXene membrane exhibited both great $\mathrm{H}_{2}$ permeability (>2200 Barrer) and high $\mathrm{H}_{2} / \mathrm{CO}_{2}$ selectivity (>160), superior to the state-of-the-art membranes (Fig. 14(a)). The high $\mathrm{H}_{2} / \mathrm{CO}_{2}$ separation selectivity is mainly attributed to the diffusion difference of gas molecules in these regular subnanometer channels.

The porous 2D nanosheets have abundant and well-defined pores, which can significantly enhance the gas permeance and selectivity. Large lateral areas of 1-nm-thick MOFs 2D nanosheets were used as building blocks to fabricate ultrathin molecular sieve membranes by hot-drop coating. ${ }^{17}$ The ultrathin porous membranes showed $\mathrm{H}_{2}$ permeance of $2700 \mathrm{GPU}$ along with a $\mathrm{H}_{2} / \mathrm{CO}_{2}$ selectivity of 291 . It is interesting to find that the disordered lamellar stacking of MOFs nanosheets could achieve a simultaneous increase in permeance and selectivity, which were higher than that of molecular sieve membranes reported to date. As the typical perforated nanosheet, single-layered porous graphene has been indeed reported with high separation performances. Through a synergistic treatment of graphene by plasma and $\mathrm{O}_{3}$ etching, Zhao et al. ${ }^{45}$ reported the preparation of high-performance single layer gas separation membranes. The membrane performance $\left(\mathrm{H}_{2}\right.$ permeance of 1340 to $6045 \mathrm{GPU}$ and $\mathrm{H}_{2} / \mathrm{CH}_{4}$ separation factor of 15.6 to 25.1 ) is one of the highest data for $\mathrm{H}_{2}$ separations.

\subsection{Liquid molecular separations}


Liquid separation, including liquid-liquid and liquid-molecular solute separations, is crucial in chemical separations and water purifications. Compared with gas separation membranes, higher stability is required for liquid separation membranes to avoid the structural deterioration in solvents.

Solvent dehydration is a costly and energy-intensive purification process, especially for the (near-) azeotropic systems. The electrophoresis deposition is an effective way to keep the good hydrophilicity of GO membrane, while narrowing down the interlayer channel size to implement size sieving between water and methanol molecules. ${ }^{34}$ Thus, the membrane could effectively separate methanol-water mixtures and exhibited high separation factor 908 (corresponding to a permeate water content of $99.89 \mathrm{wt} \%)$ and water flux $\left(1.05 \mathrm{~kg} \mathrm{~m}^{-2} \mathrm{~h}^{-1}\right)$. The methanol dehydration performance of ED-GO@PSSHF membrane resided far above the upper bound for polymeric membranes and is even comparable with A-type and T-type zeolite membranes (Fig. 14(b)). We recently reported a mixed-dimensional assembly strategy consisted of 2D COF nanosheets and 1D cellulose nanofibers (CNFs). ${ }^{48}$ The optimized membrane exhibited a flux of $8.53 \mathrm{~kg} \mathrm{~m}^{-2} \mathrm{~h}^{-1}$ with a separation factor of 3876 for $n$-butanol dehydration, a much higher performance than current polymeric, even GO and MOF membranes. Moreover, the multiple interactions between COFs and CNFs guaranteed a stable dehydration performance up to 15 days.

Membrane for organic solvent nanofiltration (OSN) or solvent-resistant nanofiltration (SRN) offers an effective recovery of the solvents from the process streams. Graphene materials have good stability in various solvents. By intercalating also cross-linking $\mathrm{SiO}_{2}$ nanoparticles into $\mathrm{GO}$ layers, a dual-spacing-channel membrane could overcome the permeance-rejection trade-off, showing a 10-times higher water permeance compared with unmodified GO membrane. The methanol permeance reached up to $290 \mathrm{~L} \mathrm{~m}^{-2} \mathrm{~h}^{-1}$ bar $^{-1}$, with more than $90 \%$ rejection of dyes with sizes larger than $1.5 \mathrm{~nm} .{ }^{19} \mathrm{COF}$ nanosheets with robust covalent bonds are ideal materials for OSN. A highly porous, self-standing COF membrane showed high permeance toward organic solvents, such as acetone $\left(194 \mathrm{~L} \mathrm{~m}^{-2} \mathrm{~h}^{-1}\right.$ bar $\left.^{-1}\right)$ and acetonitrile $\left(278 \mathrm{~L} \mathrm{~m}^{-2} \mathrm{~h}^{-1}\right.$ bar $\left.^{-1}\right){ }^{22}$ Notably, the acetonitrile flux was 2.5 orders of magnitude higher than that of reported polyamide-based nanofiltration membranes with similar solute rejection ( 99\%) ability. The membrane showed stable solvent permeance in extreme conditions (organic solvents, and mineral acids).

\subsection{Ionic separations}


Water desalination by reverse osmosis has been implemented in large-scale as the most competitive technology to meet the ever-increasing demand for freshwater. Highly efficient membranes are currently available based on thin-film composite polyamide layers and thin cellulose acetate hollow fibers. A pioneer work on graphene membranes for desalination was reported by Surwade et al. ${ }^{49}$ They prepared a single layer porous graphene membrane, by applying an oxygen plasma etching process. The membrane with well-controlled, nano-size pores exhibits a considerably rapid water flux $\left(3.6 \times 10^{6} \mathrm{~L} \mathrm{~m}^{-2} \mathrm{~h}^{-1}\right)$ and close to $100 \%$ salt rejection.

Lamellar membranes have also been reported for ionic separations. Li et al. ${ }^{31}$ reported that external pressure can accurately regulate the channel size of GO membranes (from $8.3 \AA$ to 4.5 $\AA$ ). The main rejection mechanism of the membrane changed from strongly based on the Donnan effect due to the negatively charged of the GO membranes to ion sieving, owing to the channel size. Under an external pressure of 6.0 $\mathrm{MPa}$, the membrane showed excellent performance with high rejections of $\mathrm{KCl}(92.7 \%), \mathrm{NaCl}(96.1 \%)$, and $\mathrm{CaCl}_{2}(98.3 \%)$ and high water permeance of about $22 \mathrm{~L} \mathrm{~m}^{-2} \mathrm{~h}^{-1}$ bar $^{-1}$.

Due to the hydrophilic nature, GO membrane often swell in solvents. The functionalized hydrophobic $\mathrm{MoS}_{2}$ membranes demonstrate much lower swelling compared GO. ${ }^{50}$ The channel size of the ethyl-functionalized $\mathrm{MoS}_{2}$ membrane is about $5.4 \AA$, which is smaller than the hydrated diameters of $\mathrm{Na}^{+}(7.1 \AA)$ cation. And the methyl-functionalized $\mathrm{MoS}_{2}$ membrane exhibited hydrophobic properties, contributing to the fast transport of water molecules. Manipulating the surface chemistry and the channel size therefore offers the opportunities to regulate the selectivity of the membranes while enhancing their stability. The functionalized $\mathrm{MoS}_{2}$ membranes achieved $\sim 82 \% \mathrm{NaCl}$ rejection and water permeance of $\sim 33.7 \mathrm{~L} \mathrm{~m}^{-2} \mathrm{~h}^{-1}$ bar $^{-1}$, for reverse osmosis operations, which is significantly higher than the impermeable nonfunctionalized $\mathrm{MoS}_{2}$ membrane. The desalination performances of the functionalized $\mathrm{MoS}_{2}$ membranes were superior to the previously reported RO membranes (Fig. 14(c)).

COF membranes with tuneable channel sizes and chemical functionalities can be also used for the separation of ions with a larger size. Kuehl et al. ${ }^{15}$ reported a COF-9 membrane, where a highly ordered COF was synthesized with ionizable carboxylate groups in $28 \AA$ diameter pores. The COF membrane had high rejection to cations such as tetraoctylammonium (radius = 10.9 $\AA$ ) and dodecyl (radius $=15.1 \AA$ ). Compared with a GO membrane with only $7.6 \mathrm{~L} \mathrm{~m}^{-2} \mathrm{~h}^{-1}$ bar $^{-1}$ water permeance, the highly porous COF 9 membranes exhibited a water permeance of $2260 \mathrm{~L}$ 
$\mathrm{m}^{-2} \mathrm{~h}^{-1}$ bar $^{-1}$. This work highlights the advantage of generating through-plane pores in lamellar membranes.
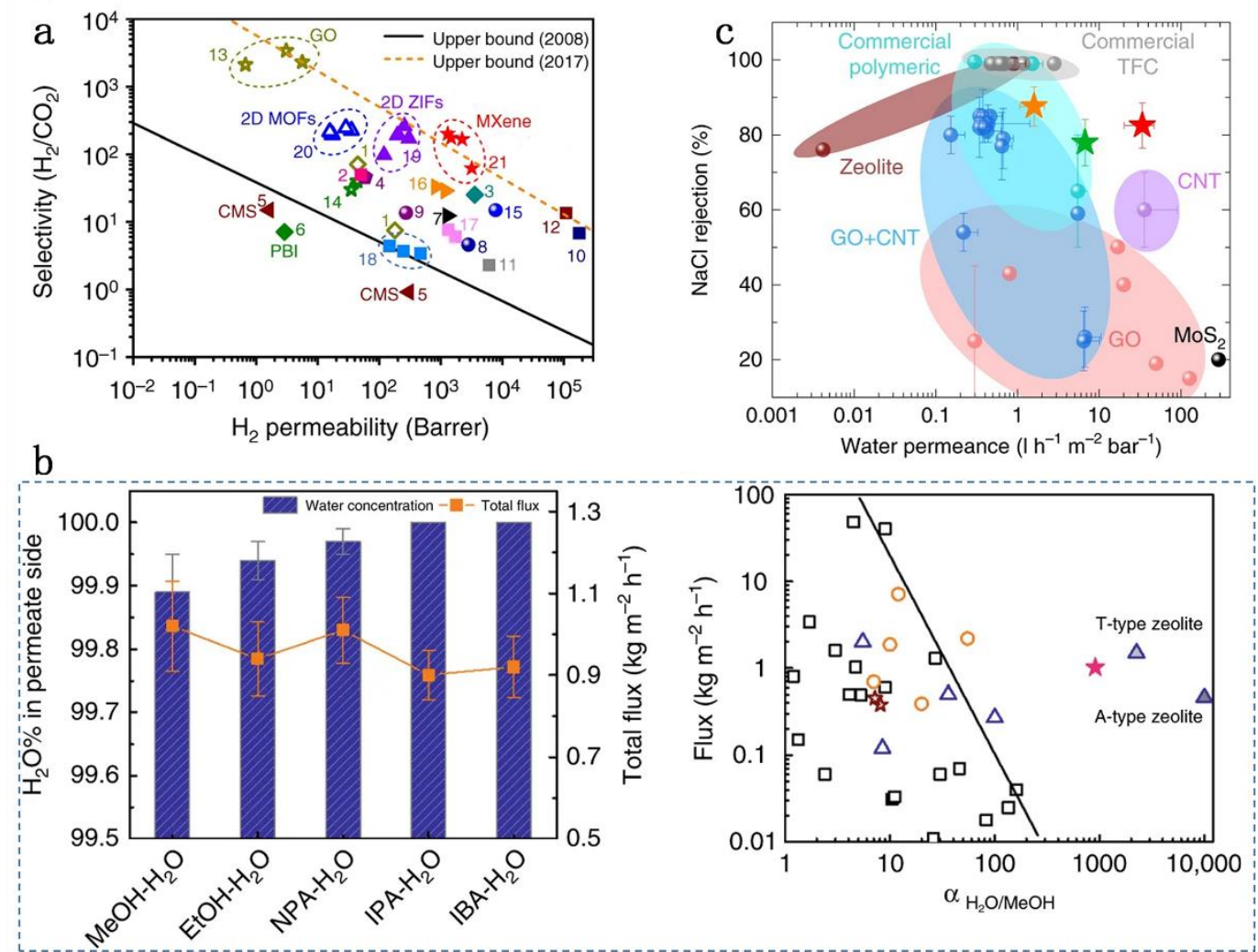

Fig. 14 Separation performance of 2DNCMs. (a) Comparison of the MXene membrane with state-of-the-art membranes for $\mathrm{H}_{2} / \mathrm{CO}_{2}$ separation. Reproduced with permission from ref. ${ }^{33}$. Copyright 2018, Springer Nature. (b) Pervaporation performance of the ED-GO@PSSHF membrane in alcohol/water mixtures (50:50 wt.\%) (left). Comparison of ED-GO@PSSHF (star mark) membrane with other membranes for the separation of methanol-water mixtures (right). Reproduced with permission from ref. ${ }^{34}$. Copyright 2017, Springer Nature. (c) Performance comparison of the 500-nm-thick functionalized $\mathrm{MoS}_{2}$ membranes (star mark) with other membranes for $\mathrm{NaCl}$ solution desalination. Reproduced with permission from ref. ${ }^{50}$. Copyright 2019, Springer Nature.

\section{Conclusion and outlook}

In summary, the emergence of various 2D nanosheets offers a great opportunity for developing ultrathin nanochannel membranes. The numerous synthesis methods have achieved a large spectrum of large-sized, single-layer nanosheets with defined pores. By adopting these nanosheets as the building blocks, 2DNCMs have been fabricated by using 
potentially scalable methods. The ideal architecture and favourable microenvironments in the nanochannels have been created to confer efficient physical or chemical-based separations. Moreover, extensive experimental exploitations have demonstrated the much higher separation perforce of 2DNCMs compared with traditional materials.

Despite the significant progress achieved during the past few years, 2DNCMs are still in the infant stage. To accelerate the research and development, the following issues need to be addressed:

(1) Materials and membrane preparations. The rapid development of chemistry and material science has spawned thousands of 2D materials. However, only a limited number have been applied in membranes. More nanosheets with novel physical or chemical structures should be attempted. On the other hand, though high separation performances have been reported, a poor repeatability is frequently seen in 2DNCMs. A major reason comes from the different properties (size, layer numbers and functional content) of the nanosheets. Therefore, the scalable, controllable synthesis methods to obtain uniform-sized, defect-free, single-layer 2D nanosheets are highly desired. Besides the material synthesis, most of membrane preparation methods for 2DNCMs are developed for flat sheets. A step forward to extend these methods to commercially applicable geometries, like spiral wound or hollow fibers, needs more research efforts.

(2) Nanochannel architecture and microenvironment. The interlayer nanochannels are often tortuous and less-efficient. Reducing membrane thickness, intercalating functional spacers, and drilling through-plane pores would be effective solutions. For lamellar membranes assembled by porous nanosheets, the undesirable channel misalignment between interlayers can be cleverly utilized to construct hierarchical channel structures (wide through-plane channels associated with narrow interlayer channels) to confer high permeance and high selectivity. Single-layer membranes based on perforated nanosheets are less feasible for practical applications but can be used to verify the simulation calculation results at fundamental level. For these membranes, the delicate pore drilling and layer transferring methods to achieve pores with high density and sub-angstrom-level precision are challenges to overcome. Chemical modification of the nanochannels may create favourable chemical microenvironments to promote the transport of targeted penetrants, which deserves more research efforts.

(3) Mass transport mechanism. The mass transport mechanisms need deeper and more extensive investigation. The mass transport through nano-confined channels exhibits different behaviours in membranes with larger pores. More experimental exploitations on fundamental 
mass transport mechanisms need to be conducted to verify the relevant simulation results, to facilitate the design of suitable membrane materials and the development of ideal membrane structure. Moreover, previous endeavours focused more on intensifying the physical separation mechanisms by tuning the channel size, the chemical separation mechanism (e.g. facilitated transport), if fully taken advantage of, may bring more promising prospects.

(4) Performance evaluation and the applications. When testing new 2D materials for membrane applications, the performance expectations can be overestimated, if only short measurements with excessively small membrane areas are reported. More uniformity would benefit future research in this field. Also, comprehensive membrane performance under industry-relevant operation conditions needs to be evaluated. Till now, the high performance of 2DNCMs was usually achieved in simulated compound mixtures, operated under mild conditions. Realistic performance losses due to the structural change within these membranes are rarely reported. A critical, honest and systematic investigation on this aspect would be highly desired, when aiming at practical applications (e.g., high temperature, organic solvents, in the presence of other impurities, long-term running). The intensively-investigated topics in polymer membranes such as plasticization, aging and fouling, should also be considered for 2DNCMs and solved in more efficient ways.

With these issues well addressed, it can be expected the 2DNCMs could break the performance limitation of conventional membranes and become next-generation membranes in molecular and ionic separations, and will play more critical roles in much broader applications relevant to energy, water, environment, foods, health, etc.

\section{Conflicts of interest}

There are no conflicts to declare

\section{Acknowledgments}

The authors are thankful for financial support from the National Natural Science Foundation of China (21621004, 21838008), National Key R\&D Program of China (2017YFB0603400), State Key Laboratory of Separation Membranes and Membrane Processes and Tianjin Polytechnic University (M1-201701), State Key Laboratory of Petroleum Pollution Control (PPC2017014), and King Abdullah University of Science and Technology (KAUST). 


\section{Notes and references}

1.

2.

3.

4.

5.

6.

7.

8.

9.

10.

11.

D. S. Sholl and R. P. Lively, Nature, 2016, 532, 435-437.

W. J. Koros and C. Zhang, Nat. Mater., 2017, 16, 289.

H. B. Park, J. Kamcev, L. M. Robeson, M. Elimelech and B. D. Freeman, Science, 2017, 356, 1137.

M. Galizia, W. S. Chi, Z. P. Smith, T. C. Merkel, R. W. Baker and B. D. Freeman, Macromolecules, 2017, 50, 7809-7843.

L. Wang, M. S. H. Boutilier, P. R. Kidambi, D. Jang, N. G. Hadjiconstantinou and R. Karnik, Nat. Nanotechnol., $2017,12,509-522$.

G. Liu, W. Jin and N. Xu, Angew. Chem. Int. Ed., 2016, 55, 13384-13397.

K. S. Novoselov, A. K. Geim, S. V. Morozov, D. Jiang, Y. Zhang, S. V. Dubonos, I. V. Grigorieva and A. A. Firsov, Science, 2004, 306, 666-669.

C. Tan, X. Cao, X.-J. Wu, Q. He, J. Yang, X. Zhang, J. Chen, W. Zhao, S. Han and G.-H. Nam, Chem. Rev., 2017, 117, 6225-6331.

S. P. Koenig, L. Wang, J. Pellegrino and J. S. Bunch, Nat. Nanotechnol., 2012, 7, 728.

H. W. Kim, H. W. Yoon, S.-M. Yoon, B. M. Yoo, B. K. Ahn, Y. H. Cho, H. J. Shin, H. Yang, U. Paik, S. Kwon, J.-Y. Choi and H. B. Park, Science, 2013, 342, 91-95.

A. Akbari, P. Sheath, S. T. Martin, D. B. Shinde, M. Shaibani, P. C. Banerjee, R. Tkacz, D. Bhattacharyya and M. Majumder, Nat. Commun., 2016, 7, 10891.

K. Celebi, J. Buchheim, R. M. Wyss, A. Droudian, P. Gasser, I. Shorubalko, J. I. Kye, C. Lee and H. G. Park, Science, 2014, 344, 289292.

Y. Yang, X. Yang, L. Liang, Y. Gao, H. Cheng, X. Li, M. Zou, R. Ma, Q. Yuan and X. J. S. Duan, Science, 2019, 364, 1057-1062.

Y. Kang, Y. Xia, H. Wang and X. Zhang, Adv. Funct. Mater., 2019, 29, 1902014.

V. A. Kuehl, J. Yin, P. H. H. Duong, B. Mastorovich, B. Newell, K. D. Li-Oakey, B. A. Parkinson and J. O. Hoberg, J. Am. Chem. Soc., 2018, 140, 18200-18207.

S. Kim, H. Wang and Y. M. Lee, Angew. Chem. Int. Ed., 2019, 58, 2-18.

Y. Peng, Y. Li, Y. Ban, H. Jin, W. Jiao, X. Liu and W. Yang, Science, 2014, 346, 1356-1359.

C. Chen, J. Wang, D. Liu, C. Yang, Y. Liu, R. S. Ruoff and W. Lei, Nat. Commun., 2018, 9, 1902.

S. Wang, D. Mahalingam, B. Sutisna and S. Nunes, J. Mater. Chem. A, 2019, 7, 11673-11682.

A. Achari, S. Sahana and M. Eswaramoorthy, Energy Environ. Sci., 2016, 9, 1224-1228.

M. Y. Jeon, D. Kim, P. Kumar, P. S. Lee, N. Rangnekar, P. Bai, M. Shete, B. Elyassi, H. S. Lee, K. Narasimharao, S. N. Basahel, S. AlThabaiti, W. Xu, H. J. Cho, E. O. Fetisov, R. Thyagarajan, R. F. DeJaco, W. Fan, K. A. Mkhoyan, J. I. Siepmann and M. Tsapatsis, Nature, 2017, 543, 690-694.

S. Kandambeth, B. P. Biswal, H. D. Chaudhari, K. C. Rout, H. S. Kunjattu, S. Mitra, S. Karak, A. Das, R. Mukherjee, U. K. Kharul and R. Banerjee, Adv. Mater., 2017, 29, 1603945.

T. Rodenas, I. Luz, G. Prieto, B. Seoane, H. Miro, A. Corma, F. Kapteijn, I. X. F. X. Llabres and J. Gascon, Nat. Mater., 2015, 14, 4855.

M. Matsumoto, L. Valentino, G. M. Stiehl, H. B. Balch, A. R. Corcos, F. Wang, D. C. Ralph, B. J. Marinas and W. R. Dichtel, Chem, 2018, 4, 308-317.

S. Huang, M. Dakhchoune, W. Luo, E. Oveisi, G. He, M. Rezaei, J. Zhao, D. T. L. Alexander, A. Zuttel, M. S. Strano and K. V. Agrawal, Nat. Commun., 2018, 9, 2632.

C. H. Tsou, Q. F. An, S. C. Lo, M. De Guzman, W. S. Hung, C. C. Hu, K. R. Lee and J. Y. Lai, J. Membr. Sci., 2015, 477, 93-100.

L. Ding, Y. Wei, Y. Wang, H. Chen, J. Caro and H. Wang, Angew. Chem. Int. Ed., 2017, 56, 1825-1829.

M. Hu and B. Mi, Environ. Sci. Technol., 2013, 47, 3715-3723.

J. Shen, G. Liu, K. Huang, Z. Chu, W. Jin and N. Xu, ACS Nano, 2016, 10, 3398-3409.

F. Zhou, H. N. Tien, W. L. Xu, J. T. Chen, Q. Liu, E. Hicks, M. Fathizadeh, S. Li and M. Yu, Nat. Commun., 2017, 8, 2107.

W. Li, W. Wu and Z. Li, ACS Nano, 2018, 12, 9309-9317.

L. Chen, G. Shi, J. Shen, B. Peng, B. Zhang, Y. Wang, F. Bian, J. Wang, D. Li, Z. Qian, G. Xu, G. Liu, J. Zeng, L. Zhang, Y. Yang, G. Zhou, M. Wu, W. Jin, J. Li and H. Fang, Nature, 2017, 550, 380-383.

L. Ding, Y. Wei, L. Li, T. Zhang, H. Wang, J. Xue, L. X. Ding, S. Wang, J. Caro and Y. Gogotsi, Nat. Commun., 2018, 9, 155

B. Qi, X. He, G. Zeng, Y. Pan, G. Li, G. Liu, Y. Zhang, W. Chen and Y. Sun, Nat. Commun., 2017, 8, 825.

W. L. Xu, C. Fang, F. Zhou, Z. Song, Q. Liu, R. Qiao and M. Yu, Nano Lett., 2017, 17, 2928-2933.

R. Nair, H. Wu, P. Jayaram, I. Grigorieva and A. Geim, Science, 2012, 335, 442-444.

S. Wang, Y. Xie, G. He, Q. Xin, J. Zhang, L. Yang, Y. Li, H. Wu, Y. Zhang, M. D. Guiver and Z. Jiang, Angew. Chem. Int. Ed., 2017, 56, 14246-14251.

B. J. S. Mi, Science, 2014, 343, 740-742.

S. Park, K.-S. Lee, G. Bozoklu, W. Cai, S. T. Nguyen and R. S. J. A. n. Ruoff, ACS Nano, 2008, 2, 572-578.

S. F. Wang, Y. Z. Wu, N. Zhang, G. W. He, Q. P. Xin, X. Y. Wu, H. Wu, X. Z. Cao, M. D. Guiver and Z. Y. Jiang, Energy Environ. Sci., 2016, 9, 3107-3112.

J. Shen, G. Z. Liu, Y. F. Ji, Q. Liu, L. Cheng, K. C. Guan, M. C. Zhang, G. P. Liu, J. Xiong, J. Yang and W. Q. Jin, Adv. Funct. Mater., 2018, 28, 1801511.

W.-S. Hung, C.-H. Tsou, M. De Guzman, Q.-F. An, Y.-L. Liu, Y.-M. Zhang, C.-C. Hu, K.-R. Lee and J.-Y. Lai, Chem. Mater., 2014, 26, 2983-2990.

K. Dey, M. Pal, K. C. Rout, H. S. Kunjattu, A. Das, R. Mukherjee, U. K. Kharul and R. Banerjee, J. Am. Chem. Soc., 2017, 139, 1308313091. 
44. H. Fan, A. Mundstock, A. Feldhoff, A. Knebel, J. Gu, H. Meng and J. Caro, J. Am. Chem. Soc., 2018, 140, $10094-10098$.

45. J. Zhao, G. He, S. Huang, L. F. Villalobos, M. Dakhchoune, H. Bassas and K. V. Agrawal, Sci. Adv., 2019, 5, eaav1851.

46. J. M. Carlsson, F. Hanke, S. Linic and M. Scheffler, Phys. Rev. Lett., 2009, 102, 166104.

47. D. Jang, J. C. Idrobo, T. Laoui and R. Karnik, ACS Nano, 2017, 11, 10042-10052.

48. H. Yang, L. Yang, H. Wang, Z. Xu, Y. Zhao, Y. Luo, N. Nasir, Y. Song, H. Wu, F. Pan and Z. Jiang, Nat. Commun., $2019,10,2101$.

49. S. P. Surwade, S. N. Smirnov, I. V. Vlassiouk, R. R. Unocic, G. M. Veith, S. Dai and S. M. Mahurin, Nat. Nanotechnol., 2015, 10, 459464.

50. L. Ries, E. Petit, T. Michel, C. C. Diogo, C. Gervais, C. Salameh, M. Bechelany, S. Balme, P. Miele and N. Onofrio, Nat. Mater., 2019, 18, 1112-1117. 